ESAIM: COCV $26(2020) 80$

https://doi.org/10.1051/cocv/2020032
ESAIM: Control, Optimisation and Calculus of Variations

www.esaim-cocv.org

\title{
ANALYSIS OF CONTROL PROBLEMS OF NONMONTONE SEMILINEAR ELLIPTIC EQUATIONS*
}

\author{
Eduardo CASAS ${ }^{1}$, Mariano Mateos ${ }^{2, * *}$ AND ARnd RÖSCH ${ }^{3}$
}

\begin{abstract}
In this paper we study optimal control problems governed by a semilinear elliptic equation. The equation is nonmonotone due to the presence of a convection term, despite the monotonocity of the nonlinear term. The resulting operator is neither monotone nor coervive. However, by using conveniently a comparison principle we prove existence and uniqueness of solution for the state equation. In addition, we prove some regularity of the solution and differentiability of the relation control-to-state. This allows us to derive first and second order conditions for local optimality.
\end{abstract}

Mathematics Subject Classification. 35J61, 49J20, 49K20.

Received June 13, 2019. Accepted May 25, 2020.

\section{INTRODUCTION}

In this paper, we consider an optimal control problem associated with the following elliptic semilinear equation

$$
\left\{\begin{array}{l}
A y+b(x) \cdot \nabla y+f(x, y)=u \text { in } \Omega \\
y=0 \text { on } \Gamma
\end{array}\right.
$$

where $A$ is an elliptic operator, $b: \Omega \longrightarrow \mathbb{R}^{n}$ is a given function, $f: \Omega \times \mathbb{R} \longrightarrow \mathbb{R}$ is nondecreasing monotone in the second variable, $u \in L^{2}(\Omega), \Omega$ is a domain in $\mathbb{R}^{n}, n=2$ or 3 , and $\Gamma$ is the boundary of $\Omega$. The precise assumptions on these data will be given in the next section. Due to the convection term induced by $b$, the linear part of the above operator is nonmonote. We emphasize that here we neither assume that $\operatorname{div} b=0$ nor $b$ is small. Consequently, the bilinear form associated with the linear part of the operator is not necessarily coercive. This introduces some important difficulties in the analysis of the equation. A thorough study is needed to prove existence and uniqueness of a solution of the equation (1.1) for every $u$. This study makes a strong use of a comparison principle.

In many publications $\operatorname{div} b=0$ is assumed. This property is satisfied in several applications, for instance if the quantity $b$ represents a velocity field of an incompressible Navier-Stokes flow. If the flow is compressible, the

\footnotetext{
* The first two authors were partially supported by Spanish Ministerio de Economía y Competitividad under research project MTM2017-83185-P

Keywords and phrases: Optimal control, semilinear partial differential equation, optimality conditions.

${ }^{1}$ Departmento de Matemática Aplicada y Ciencias de la Computación, E.T.S.I. Industriales y de Telecomunicación, Universidad de Cantabria, 39005 Santander, Spain.

2 Departamento de Matemáticas, Campus de Gijón, Universidad de Oviedo, 33203 Gijón, Spain.

3 Fakultät für Mathematik, Universtät Duisburg-Essen, 45127 Essen, Germany.

** Corresponding author: mmateos@uniovi.es
} 
assumption $\operatorname{div} b=0$ cannot be justified. Some examples of applications where the divergence of the convection term need not be zero can be found in the introductory chapter of the book [1].

In many books devoted to partial differential equations, the convection term appears and it is not assumed to have zero divergence. Let us mention the classical books [12] or [16]; see also [18]. However, only a few references treat the topic of existence and uniqueness of solution for linear elliptic equations with convection term such that $\operatorname{div} b \neq 0$ and $b$ is not small. When the nonlinear term $f$ is not present in the state equation, the reader is referred to the early reference [19] for the existence and uniqueness of a solution; see also Theorem 8.3 in [13] or the recent reference [4]. In [5], the case of a semilinear equation in dimension $n \geq 3$ with the non-linearity $y|y|^{\lambda-1}, \lambda>\frac{n}{n-2}$, is studied. As far as we know, the most general and complete results for the analysis of equation (1.1) are the ones presented below in Section 2.

The case of nonmonotone quasilinear elliptic equations was considered in [8] and [15]. However, in the last two papers, the operator was coercive. The equation considered in this paper does not fit in the problems studied in the mentioned references.

Associated with the state equation (1.1) we consider the following control problem:

$$
\text { (P) } \min _{u \in U_{\text {ad }}} J(u):=\int_{\Omega} L\left(x, y_{u}(x)\right) \mathrm{d} x+\frac{\nu}{2} \int_{\Omega} u^{2}(x) \mathrm{d} x
$$

where $y_{u}$ is the solution of (1.1) associated with $u, L: \Omega \times \mathbb{R} \longrightarrow \mathbb{R}$ is a given function, $\nu>0$, and

$$
U_{\text {ad }}=\left\{u \in L^{2}(\Omega): \alpha \leq u(x) \leq \beta \text { for a.a. } x \in \Omega\right\}
$$

with $-\infty \leq \alpha<\beta \leq+\infty$. A precise analysis of the state equation allows us to prove the existence of a solution for $(\mathrm{P})$ as well as to get the first and second order optimality conditions.

Typical examples of nonlinearities in the state equations are $f(x, y)=a_{0}(x)|y|^{r} y$ with $r>0$ or $f(x, y)=$ $a_{0}(x) \exp (y)$, where $a_{0}$ is assumed to be nonnegative and bounded. The assumption $r>1$ is needed to prove the existence of a second derivative of $J$. Concerning the functional $J$, the usual tracking cost functional falls into this framework by setting $L(x, y)=\frac{1}{2}\left(y-y_{d}(x)\right)^{2}$ for some fixed function $y_{d} \in L^{2}(\Omega)$.

To our best knowledge, this is the first time that a control problem governed by a nonmonotone and noncoercive equation of the kind described here has been considered. The methods to study the control problem are technically more involved than those used for problems governed by coercive equations, as it can be seen not only in the study of the state equation, but also in the proofs of some results such as Lemma 3.5 or Theorem 3.8.

The paper is organized as follows. In Section 2, the state equation is analyzed. We address the issues of existence, uniqueness and regularity results of the solution for both the linear and semilinear cases. Differentiability of the relation control-to-state is also established. Finally, the existence of solution for (P) as well as first and second order optimality conditions are proved in Section 3. Based on the results established in this paper, the numerical analysis for $(\mathrm{P})$ will be carried out in a forthcoming paper.

\section{Analysis of the state Equation}

In this section we study the equation (1.1) proving some results that will be used in the analysis of the control problem (P). Before studying (1.1), we analyze a linear equation involving the convection term. The section is divided into two subsections. The first one is devoted to the linear equation and the second to the study of (1.1)

\subsection{Study of the linear operator}

The following assumption is needed for this analysis. 
Assumption 1. $\Omega$ is an open domain in $\mathbb{R}^{n}, n=2$ or 3 , with a Lipschitz boundary $\Gamma$. $A$ is the operator given by

$$
A y=-\sum_{i, j=1}^{n} \partial_{x_{j}}\left(a_{i j}(x) \partial_{x_{i}} y\right) \text { with } a_{i j} \in L^{\infty}(\Omega)
$$

and satisfying the following ellipticity condition:

$$
\exists \Lambda>0 \text { such that } \sum_{i, j=1}^{n} a_{i j}(x) \xi_{i} \xi_{j} \geq \Lambda|\xi|^{2} \quad \forall \xi \in \mathbb{R}^{n} \text { and for a.a. } x \in \Omega \text {. }
$$

The function $b: \Omega \rightarrow \mathbb{R}^{n}$ satisfies $b \in L^{p}(\Omega)^{n}$ with $p \geq 3$ if $n=3$ and $p>2$ if $n=2$. For the function $a_{0}: \Omega \rightarrow \mathbb{R}$ it is assumed that $a_{0} \in L^{q}(\Omega)$ with $q>1$ if $n=2$ and $q \geq \frac{3}{2}$ if $n=3$.

Unless stated otherwise, in the rest of the paper $\bar{p}$ will denote a number such that $\bar{p}>n / 2$.

Notice that with this choice, $L^{\bar{p}}(\Omega) \subset W^{-1, r}(\Omega) \subset H^{-1}(\Omega)$ for some $r>n$.

Along this paper we will take

$$
\|y\|_{H_{0}^{1}(\Omega)}=\left(\int_{\Omega}|\nabla y(x)|^{2} \mathrm{~d} x\right)^{\frac{1}{2}}
$$

From the Poincaré inequality and the Sobolev embedding theorem, we know that there exist two constants $C_{\Omega}$ and $K_{\Omega}$ such that

$$
\|y\|_{L^{2}(\Omega)} \leq C_{\Omega}\|y\|_{H_{0}^{1}(\Omega)} \quad \text { and } \quad\|y\|_{L^{6}(\Omega)} \leq K_{\Omega}\|y\|_{H_{0}^{1}(\Omega)} \quad \forall y \in H_{0}^{1}(\Omega)
$$

As a consequence, we have that $\|y\|_{H^{-1}(\Omega)} \leq C_{\Omega}\|y\|_{L^{2}(\Omega)}$ for all $y \in L^{2}(\Omega)$.

Let us consider the elliptic operator

$$
\mathcal{A} y=A y+b(x) \cdot \nabla y+a_{0}(x) y \text { with } a_{0} \geq 0 .
$$

We first prove continuity of this operator and Gårding's inequality.

Lemma 2.1. Under Assumption 1 we have that $\mathcal{A} \in \mathcal{L}\left(H_{0}^{1}(\Omega), H^{-1}(\Omega)\right)$ and there exists a constant $C_{\Lambda, b}$ such that

$$
\langle\mathcal{A} z, z\rangle_{H^{-1}(\Omega), H_{0}^{1}(\Omega)} \geq \frac{\Lambda}{4}\|z\|_{H_{0}^{1}(\Omega)}^{2}-C_{\Lambda, b}\|z\|_{L^{2}(\Omega)}^{2} \quad \forall z \in H_{0}^{1}(\Omega) .
$$

Proof. Let us show that $\mathcal{A}$ is a linear continuous operator. We will prove the result for dimension $n=3$ and we can argue in a similar way for dimension $n=2$. It is obvious that $A: H_{0}^{1}(\Omega) \longrightarrow H^{-1}(\Omega)$ is a continuous linear mapping due to the fact that $a_{i j} \in L^{\infty}(\Omega)$. Moreover, from (2.1) and Hölder inequality we infer for every $z \in H_{0}^{1}(\Omega)$

$$
\begin{aligned}
& \|b \cdot \nabla z\|_{L^{\frac{6}{5}}(\Omega)} \leq\|b\|_{L^{3}(\Omega)^{3}}\|\nabla z\|_{L^{2}(\Omega)^{3}}=\|b\|_{L^{3}(\Omega)^{3}}\|z\|_{H_{0}^{1}(\Omega)}, \\
& \left\|a_{0} z\right\|_{L^{\frac{6}{5}}(\Omega)} \leq\left\|a_{0}\right\|_{L^{\frac{3}{2}}(\Omega)}\|z\|_{L^{6}(\Omega)} \leq K_{\Omega}\left\|a_{0}\right\|_{L^{\frac{3}{2}}(\Omega)}\|z\|_{H_{0}^{1}(\Omega)} .
\end{aligned}
$$

Hence, we have that $\mathcal{A}$ is a well-posed linear and continuous operator. 
Let us prove (2.3). In this case, the proofs for $n=3$ and $n=2$ are slightly different. We start with $n=2$. Using that $a_{0} \geq 0$ and Young and Hölder inequalities we get

$$
\begin{aligned}
\langle\mathcal{A} z, z\rangle_{H^{-1}(\Omega), H_{0}^{1}(\Omega)} & \geq \Lambda\|\nabla z\|_{L^{2}(\Omega)^{n}}^{2}-\|\nabla z\|_{L^{2}(\Omega)^{n}}\|b z\|_{L^{2}(\Omega)^{n}} \geq \frac{\Lambda}{2}\|\nabla z\|_{L^{2}(\Omega)^{n}}^{2}-\frac{1}{2 \Lambda}\|b z\|_{L^{2}(\Omega)^{n}}^{2} \\
& \geq \frac{\Lambda}{2}\|\nabla z\|_{L^{2}(\Omega)^{n}}^{2}-\frac{1}{2 \Lambda}\|b\|_{L^{p}(\Omega)^{n}}^{2}\|z\|_{L^{\frac{2 p}{p-2}(\Omega)}}^{2}
\end{aligned}
$$

Observe that the assumption $p>2$ implies that $2<\frac{2 p}{p-2}<\infty$ if $n=2$. Now, we apply Lions' Lemma ([17], Chap. 2, Lem. 6.1), to the spaces $H_{0}^{1}(\Omega) \subset L^{\frac{2 p}{p-2}}(\Omega) \subset L^{2}(\Omega)$ to deduce the existence of a constant $C_{0}$ depending of $\Lambda$ and $\|b\|_{L^{p}(\Omega)^{n}}$ such that

$$
\|z\|_{L^{\frac{2 p}{p-2}}(\Omega)} \leq \frac{\Lambda}{2\|b\|_{L^{p}(\Omega)^{n}}}\|\nabla z\|_{L^{2}(\Omega)^{n}}+C_{0}\|z\|_{L^{2}(\Omega)}
$$

From the last two inequalities we conclude (2.3) with

$$
C_{\Lambda, b}=\frac{C_{0}^{2}\|b\|_{L^{p}(\Omega)^{n}}^{2}}{\Lambda}
$$

For $n=3$ we proceed as follows. From Lemma 3.1 in [18], we know that, for any $\varepsilon>0$ there exists a constant $K_{\varepsilon, b}>0$ depending on $b$ and $\varepsilon$ such that $b=b^{\prime}+b^{\prime \prime}$, with $\left\|b^{\prime}\right\|_{L^{\infty}(\Omega)^{n}}<K_{\varepsilon, b}$ and $\left\|b^{\prime \prime}\right\|_{L^{3}(\Omega)^{n}}<\varepsilon$. Taking $\varepsilon=\Lambda /\left(4 K_{\Omega}\right), K_{\Omega}$ satisfying (2.1), and using that $a_{0} \geq 0$, Hölder and Young inequalities and (2.1), we obtain

$$
\begin{aligned}
\langle\mathcal{A} z, z\rangle_{H^{-1}(\Omega), H_{0}^{1}(\Omega)} & \geq \Lambda\|\nabla z\|_{L^{2}(\Omega)^{n}}^{2}-\int_{\Omega}\left(b^{\prime}+b^{\prime \prime}\right) \cdot \nabla z z \mathrm{~d} x \\
& \geq \Lambda \mid \nabla z\left\|_{L^{2}(\Omega)^{n}}^{2}-\right\| b^{\prime}\left\|_{L^{\infty}(\Omega)^{n}}\right\| \nabla z\left\|_{L^{2}(\Omega)^{n}}\right\| z\left\|_{L^{2}(\Omega)}-\right\| b^{\prime \prime}\left\|_{L^{3}(\Omega)^{n}}\right\| \nabla z\left\|_{L^{2}(\Omega)^{n}}\right\| z \|_{L^{6}(\Omega)} \\
& \geq \Lambda\|\nabla z\|_{L^{2}(\Omega)^{n}}^{2}-\frac{\Lambda}{2}\|\nabla z\|_{L^{2}(\Omega)^{n}}^{2}-\frac{K_{\varepsilon, b}^{2}}{2 \Lambda}\|z\|_{L^{2}(\Omega)}^{2}-\frac{\Lambda}{4}\|\nabla z\|_{L^{2}(\Omega)^{n}}^{2}
\end{aligned}
$$

and (2.3) follows with a constant $C_{\Lambda, b}=K_{\varepsilon, b}^{2} /(2 \Lambda)$.

Theorem 2.2. Under Assumption 1, the linear operator $\mathcal{A}: H_{0}^{1}(\Omega) \longrightarrow H^{-1}(\Omega)$ is an isomorphism.

This theorem can be deduced from the results in [19]. We include a direct proof for the convenience of the reader.

Proof. From Lemma 2.1 we know that $\mathcal{A}$ is a well-posed linear and continuous operator. Let us divide the proof into three steps.

Step 1. $\mathcal{A}$ is injective. We make this proof for $n=3$. The case $n=2$ follows along the same lines with minor changes. To prove that the kernel of $\mathcal{A}$ is reduced to 0 we adapt the proof of Theorem 8.1 in [13]. Let $y \in H_{0}^{1}(\Omega)$ satisfy that $\mathcal{A} y=0$. We prove that $y \leq 0$ in $\Omega$, the contrary inequality follows by arguing on $-y$. We argue by contradiction and we suppose that this is false. Then, we take $0<\rho<\operatorname{ess} \sup _{x \in \Omega} y(x) \leq+\infty$ and we define $y_{\rho}(x)=(y(x)-\rho)^{+}$. Obviously we have that $y_{\rho} \in H_{0}^{1}(\Omega)$. We denote $\Omega_{\rho}=\left\{x \in \Omega: \nabla y_{\rho}(x) \neq 0\right\}$, then

$$
\nabla y_{\rho}(x)=\left\{\begin{array}{cl}
\nabla y(x) & \text { if } y(x)>\rho, \\
0 & \text { otherwise, }
\end{array} \quad \text { and } y_{\rho}(x)=0 \text { if } y(x) \leq \rho .\right.
$$


Using these facts and our assumptions on $b$ and $a_{0}$ we get

$$
\begin{aligned}
0 & =\int_{\Omega}\left(\sum_{i, j=1}^{n} a_{i j}(x) \partial_{x_{i}} y \partial_{x_{j}} y_{\rho}+[b(x) \cdot \nabla y] y_{\rho}+a_{0}(x) y y_{\rho}\right) \mathrm{d} x \\
& \geq \int_{\Omega_{\rho}}\left(\sum_{i, j=1}^{n} a_{i j}(x) \partial_{x_{i}} y_{\rho} \partial_{x_{j}} y_{\rho}+\left[b(x) \cdot \nabla y_{\rho}\right] y_{\rho}\right) \mathrm{d} x \\
& \geq \Lambda\left\|\nabla y_{\rho}\right\|_{L^{2}\left(\Omega_{\rho}\right)^{n}}^{2}-\|b\|_{L^{3}\left(\Omega_{\rho}\right)^{n}}\left\|\nabla y_{\rho}\right\|_{L^{2}\left(\Omega_{\rho}\right)^{n}}\left\|y_{\rho}\right\|_{L^{6}\left(\Omega_{\rho}\right)} .
\end{aligned}
$$

From here and (2.1) we infer

$$
\begin{aligned}
& \left\|y_{\rho}\right\|_{L^{6}\left(\Omega_{\rho}\right)} \leq\left\|y_{\rho}\right\|_{L^{6}(\Omega)} \leq K_{\Omega}\left\|\nabla y_{\rho}\right\|_{L^{2}(\Omega)^{n}} \\
& =K_{\Omega}\left\|\nabla y_{\rho}\right\|_{L^{2}\left(\Omega_{\rho}\right)^{n}} \leq \frac{1}{\Lambda} K_{\Omega}\|b\|_{L^{3}\left(\Omega_{\rho}\right)^{n}}\left\|y_{\rho}\right\|_{L^{6}\left(\Omega_{\rho}\right)} .
\end{aligned}
$$

Hence, we have

$$
\|b\|_{L^{3}\left(\Omega_{\rho}\right)^{n}} \geq \frac{\Lambda}{K_{\Omega}}>0
$$

which contradicts the fact that $\left|\Omega_{\rho}\right| \rightarrow 0$ if $\rho \rightarrow \operatorname{ess}_{\sup _{x \in \Omega}} y(x)$. Indeed, if the set of points $E=\{x \in \Omega: y(x)=$

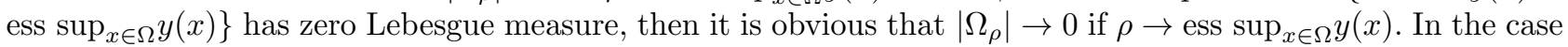
that $|E|>0$, then we have that $\nabla y(x)=0$ a.e. in $E$ ([13], Lem. 7.7) and consequently $\nabla y_{\rho}(x)=\nabla y(x)=0$ a.e. in $E$ as well. Hence, $\left|\Omega_{\rho}\right| \rightarrow 0$ holds in any case.

We notice that the same procedure can be used to prove the injectivity of $\mathcal{A}^{*}: H_{0}^{1}(\Omega) \rightarrow H^{-1}(\Omega)$, given by

$$
\mathcal{A}^{*} \varphi=A^{*} \varphi-\operatorname{div}(\varphi b(x))+a_{0} \varphi .
$$

To do this, we take $\varphi \in H_{0}^{1}(\Omega)$ such that $\mathcal{A}^{*} \varphi=0$, and define for all $\varepsilon \geq 0, \Omega^{\varepsilon}=\{x \in \Omega:|\varphi(x)|>\varepsilon\}$ and $\varphi^{\varepsilon}(x)=\operatorname{proj}_{[-\varepsilon, \varepsilon]}(\varphi(x))$. Using integration by parts, that $a_{0}(x) \varphi(x) \varphi^{\varepsilon}(x) \geq 0$ and the fact that $\nabla \varphi^{\varepsilon}=0$ in $\Omega^{\varepsilon}$, we have

$$
\begin{aligned}
0 & =\int_{\Omega}\left(\sum_{i, j=1}^{n} a_{i j}(x) \partial_{x_{i}} \varphi \partial_{x_{j}} \varphi^{\varepsilon}-\operatorname{div}(\varphi b(x)) \varphi^{\varepsilon}+a_{0}(x) \varphi \varphi^{\varepsilon}\right) \mathrm{d} x \\
& =\int_{\Omega}\left(\sum_{i, j=1}^{n} a_{i j}(x) \partial_{x_{i}} \varphi \partial_{x_{j}} \varphi^{\varepsilon}+\varphi b(x) \cdot \nabla \varphi^{\varepsilon}+a_{0}(x) \varphi \varphi^{\varepsilon}\right) \mathrm{d} x \\
& \geq \Lambda\left\|\nabla \varphi^{\varepsilon}\right\|_{L^{2}(\Omega)^{n}}^{2}-\|b\|_{L^{3}\left(\Omega^{0} \backslash \Omega^{\varepsilon}\right)^{n}}\left\|\nabla \varphi^{\varepsilon}\right\|_{L^{2}(\Omega)^{n}}\left\|\varphi^{\varepsilon}\right\|_{L^{6}\left(\Omega^{0} \backslash \Omega^{\varepsilon}\right)} .
\end{aligned}
$$

From here we infer that

$$
\left\|\nabla \varphi^{\varepsilon}\right\|_{L^{2}(\Omega)^{n}} \leq \frac{1}{\Lambda}\|b\|_{L^{3}\left(\Omega^{0} \backslash \Omega^{\varepsilon}\right)^{n}}\left\|\varphi^{\varepsilon}\right\|_{L^{6}\left(\Omega^{0} \backslash \Omega^{\varepsilon}\right)} \leq \frac{1}{\Lambda} \varepsilon\left|\Omega^{0} \backslash \Omega^{\varepsilon}\right|^{\frac{1}{6}}\|b\|_{L^{3}\left(\Omega^{0} \backslash \Omega^{\varepsilon}\right)^{n}} .
$$

Using this and (2.1) we get

$$
\left|\Omega^{\varepsilon}\right|=\frac{1}{\varepsilon^{2}} \int_{\Omega^{\varepsilon}} \varphi^{\varepsilon}(x)^{2} \mathrm{~d} x \leq \frac{1}{\varepsilon^{2}} \int_{\Omega} \varphi^{\varepsilon}(x)^{2} \mathrm{~d} x \leq \frac{1}{\varepsilon^{2}} C_{\Omega}^{2}\left\|\nabla \varphi^{\varepsilon}\right\|_{L^{2}(\Omega)^{n}}^{2} \leq \frac{C_{\Omega}^{2}}{\Lambda^{2}}\left|\Omega^{0} \backslash \Omega^{\varepsilon}\right|^{\frac{1}{3}}\|b\|_{L^{3}\left(\Omega^{0} \backslash \Omega^{\varepsilon}\right)^{n}}^{2},
$$


and $\left|\Omega^{0}\right|=\lim _{\varepsilon \rightarrow 0}\left|\Omega^{\varepsilon}\right|=0$ and, hence, $\varphi=0$.

Step 2. The range of $\mathcal{A}$ is dense and closed. To see that it is dense, we argue by contradiction: suppose it is not dense. Then, there exists $z \in H_{0}^{1}(\Omega)$ with $z \neq 0$ such that $\langle\mathcal{A} y, z\rangle_{H^{-1}(\Omega), H_{0}^{1}(\Omega)}=0$ for all $y \in H_{0}^{1}(\Omega)$. By duality, this implies $\left\langle\mathcal{A}^{*} z, y\right\rangle_{H^{-1}(\Omega), H_{0}^{1}(\Omega)}=0$ for all $y \in H_{0}^{1}(\Omega)$, and hence $\mathcal{A}^{*} z=0$. Since $\mathcal{A}^{*}$ is injective, we obtain that $z=0$, which is a contradiction.

Let us check that the range of $\mathcal{A}$ is closed. Let $\left\{f_{k}\right\}_{k=1}^{\infty}$ be a sequence in the range of $\mathcal{A}$ such that $f_{k} \rightarrow f$ in $H^{-1}(\Omega)$. Let $y_{k} \in H_{0}^{1}(\Omega)$ be such that $\mathcal{A} y_{k}=f_{k}$ for every $k \geq 1$. We are going to prove that $\left\{y_{k}\right\}_{k=1}^{\infty}$ converges weakly in $H_{0}^{1}(\Omega)$ to some element $y \in H_{0}^{1}(\Omega)$ satisfying $\mathcal{A} y=f$.

First, let us prove that $y_{k}$ is bounded in $L^{2}(\Omega)$. We argue by contradiction. Suppose it is not. Then, for a subsequence denoted in the same form, we have that $\left\|y_{k}\right\|_{L^{2}(\Omega)} \rightarrow+\infty$. Define $\hat{y}_{k}=y_{k} /\left\|y_{k}\right\|_{L^{2}(\Omega)}$, and $\hat{f}_{k}=f_{k} /\left\|y_{k}\right\|_{L^{2}(\Omega)}$. We have that $\mathcal{A} \hat{y}_{k}=\hat{f}_{k}$. Using again Gårding's inequality and the fact that $\left\|\hat{y}_{k}\right\|_{L^{2}(\Omega)}=1$, we obtain

$$
\begin{aligned}
\frac{\Lambda}{4}\left\|\hat{y}_{k}\right\|_{H_{0}^{1}(\Omega)}^{2} & \leq\left\langle\mathcal{A} \hat{y}_{k}, \hat{y}_{k}\right\rangle_{H^{-1}(\Omega), H_{0}^{1}(\Omega)}+C_{\Lambda, b}\left\|\hat{y}_{k}\right\|_{L^{2}(\Omega)}^{2}=\left\langle\hat{f}_{k}, \hat{y}_{k}\right\rangle_{H^{-1}(\Omega), H_{0}^{1}(\Omega)}+C_{\Lambda, b}\left\|\hat{y}_{k}\right\|_{L^{2}(\Omega)}^{2} \\
& \leq\left\|\hat{f}_{k}\right\|_{H^{-1}(\Omega)}\left\|\hat{y}_{k}\right\|_{H_{0}^{1}(\Omega)}+C_{\Lambda, b} \leq \frac{2}{\Lambda}\left\|\hat{f}_{k}\right\|_{H^{-1}(\Omega)}^{2}+\frac{\Lambda}{8}\left\|\hat{y}_{k}\right\|_{H_{0}^{1}(\Omega)}^{2}+C_{\Lambda, b},
\end{aligned}
$$

and therefore

$$
\frac{\Lambda}{8}\left\|\hat{y}_{k}\right\|_{H_{0}^{1}(\Omega)}^{2} \leq \frac{2}{\Lambda}\left\|\hat{f}_{k}\right\|_{H^{-1}(\Omega)}^{2}+C_{\Lambda, b} .
$$

Since $\left\{f_{k}\right\}_{k=1}^{\infty}$ is bounded in $H^{-1}(\Omega)$, we infer from the above inequality that $\left\{\hat{y}_{k}\right\}_{k=1}^{\infty}$ is bounded in $H_{0}^{1}(\Omega)$. Hence, there exists $\hat{y} \in H_{0}^{1}(\Omega)$ such that (for a new subsequence, again denoted in the same way) $\hat{y}_{k} \rightarrow \hat{y}$ in $H_{0}^{1}(\Omega)$, and by Rellich's theorem $\hat{y}_{k} \rightarrow \hat{y}$ in $L^{2}(\Omega)$. In particular, this implies that $\|\hat{y}\|_{L^{2}(\Omega)}=1$. On the other hand, using that $\left\|\hat{f}_{k}\right\|_{H^{-1}(\Omega)} \rightarrow 0$ and $\mathcal{A} \hat{y}_{k}=\hat{f}_{k}$, we can pass to the limit in this equation and deduce that $\mathcal{A} \hat{y}=0$. Hence $\hat{y}=0$ and we have a contradiction. Thus, $\left\{y_{k}\right\}_{k=1}^{\infty}$ is bounded in $L^{2}(\Omega)$.

Using again Gårding's inequality for the equation $\mathcal{A} y_{k}=f_{k}$, we obtain as above that

$$
\frac{\Lambda}{8}\left\|y_{k}\right\|_{H_{0}^{1}(\Omega)}^{2} \leq \frac{2}{\Lambda}\left\|f_{k}\right\|_{H^{-1}(\Omega)}^{2}+C_{\Lambda, b}\left\|y_{k}\right\|_{L^{2}(\Omega)}^{2}
$$

and therefore, $\left\{y_{k}\right\}_{k=1}^{\infty}$ is bounded in $H_{0}^{1}(\Omega)$. So there exists $y \in H_{0}^{1}(\Omega)$ and a subsequence, denoted in the same way, such that $y_{k} \rightarrow y$ in $H_{0}^{1}(\Omega)$. Taking the limit in the equation $\mathcal{A} y_{k}=f_{k}$, we have that $\mathcal{A} y=f$. Hence, $f$ belongs to the range of $\mathcal{A}$ and this subspace is closed in $H_{0}^{1}(\Omega)$.

The following corollary is a straightforward application of Theorem 2.2 and the Hölder regularity result ([18], Thm. 7.3); see also Theorem 14.1 in [16] and the remark after it.

Corollary 2.3. Suppose Assumption 1 holds. Then, for every $u \in L^{\bar{p}}(\Omega)$ there exists a unique function $y \in$ $H_{0}^{1}(\Omega) \cap C^{0, \mu}(\bar{\Omega})$, for some $\mu \in(0,1)$ independent of $u$, satisfying $\mathcal{A} y=u$. Moreover, there exists a constant $C_{\mathcal{A}, \mu}$ such that

$$
\|y\|_{C^{0, \mu}(\bar{\Omega})} \leq C_{\mathcal{A}, \mu}\|u\|_{L^{\bar{p}}(\Omega)} \quad \forall u \in L^{\bar{p}}(\Omega) .
$$

The adjoint operator also enjoys these properties. 
Corollary 2.4. Under Assumption 1, the adjoint operator $\mathcal{A}^{*}: H_{0}^{1}(\Omega) \longrightarrow H^{-1}(\Omega)$ given by

$$
\mathcal{A}^{*} \varphi=A^{*} \varphi-\operatorname{div}[b(x) \varphi]+a_{0}(x) \varphi
$$

is an isomorphism. Moreover, for every $f \in L^{\bar{p}}(\Omega)$, there exists a unique $\varphi \in H_{0}^{1}(\Omega)$ satisfying $\mathcal{A}^{*} \varphi=f$ and there exist $\mu \in(0,1)$ and $C_{\mathcal{A}^{*}, \mu}$ independent of $f$ such that $\varphi \in C^{0, \mu}(\bar{\Omega})$ and

$$
\|\varphi\|_{C^{0, \mu}(\bar{\Omega})} \leq C_{\mathcal{A}^{*}, \mu}\|f\|_{L^{\bar{p}}(\Omega)} \quad \forall f \in L^{\bar{p}}(\Omega) .
$$

Proof. The first statement follows directly from Theorem 2.2. The second is again a consequence of ([18], Thm. 7.3 or [16], Thm. 14.1).

Under additional assumptions we have the following regularity result.

Theorem 2.5. Suppose Assumption 1 holds and assume further that $a_{i j} \in C^{0,1}(\bar{\Omega})$ for $1 \leq i, j \leq n, a_{0} \in L^{2}(\Omega)$ and $\Gamma$ is of class $C^{1,1}$ or $\Omega$ is convex. Then, $\mathcal{A}: H^{2}(\Omega) \cap H_{0}^{1}(\Omega) \longrightarrow L^{2}(\Omega)$ is an isomorphism.

Proof. First we observe that $\mathcal{A}: H^{2}(\Omega) \cap H_{0}^{1}(\Omega) \longrightarrow L^{2}(\Omega)$ is an injective, continuous linear operator. Indeed, taking into account that $H^{2}(\Omega) \subset W^{1, \frac{2 p}{p-2}}(\Omega)$ and $H^{2}(\Omega) \subset C(\bar{\Omega})$, we get the following estimates

$$
\begin{aligned}
& \left\|\partial_{x_{j}}\left(a_{i j} \partial_{x_{i}} y\right)\right\|_{L^{2}(\Omega)} \leq\left\|\partial_{x_{j}} a_{i j} \partial_{x_{i}} y\right\|_{L^{2}(\Omega)}+\left\|a_{i j} \partial_{x_{i}, x_{j}}^{2} y\right\|_{L^{2}(\Omega)} \\
& \leq\left\|\partial_{x_{j}} a_{i j}\right\|_{L^{\infty}(\Omega)}\left\|\partial_{x_{i}} y\right\|_{L^{2}(\Omega)}+\left\|a_{i j}\right\|_{L^{\infty}(\Omega)}\left\|\partial_{x_{i}, x_{j}}^{2} y\right\|_{L^{2}(\Omega)} \leq\left\|a_{i j}\right\|_{C^{0,1}(\bar{\Omega})}\|y\|_{H^{2}(\Omega)}, \\
& \|b \cdot \nabla y\|_{L^{2}(\Omega)} \leq\|b\|_{L^{p}(\Omega)^{n}}\|\nabla y\|_{L^{\frac{2 p}{p-2}(\Omega)^{n}}} \leq C\|b\|_{L^{p}(\Omega)^{n}}\|y\|_{H^{2}(\Omega)}, \\
& \left\|a_{0} y\right\|_{L^{2}(\Omega)} \leq\left\|a_{0}\right\|_{L^{2}(\Omega)}\|y\|_{L^{\infty}(\Omega)} \leq C\left\|a_{0}\right\|_{L^{2}(\Omega)}\|y\|_{H^{2}(\Omega)} .
\end{aligned}
$$

The above estimates prove that $\mathcal{A}$ is well defined and continuous. The injectivity of $\mathcal{A}$ is an immediate consequence of Theorem 2.2. Let us prove that $\mathcal{A}$ is surjective. Given $u \in L^{2}(\Omega)$ arbitrary, from Theorem 2.2 we deduce the existence of an element $y \in H_{0}^{1}(\Omega)$ such that $\mathcal{A} y=u$. We have to prove that $y \in H^{2}(\Omega)$. To this end we divide the proof into three steps.

Step 1. Here we regularize the coefficients $b$ and $a_{0}$. We make the proof for $n=3$ and comment later the modifications for $n=2$.

The Lipschitz regularity of the coefficients $a_{i j}$ implies that $A: H^{2}(\Omega) \cap H_{0}^{1}(\Omega) \longrightarrow L^{2}(\Omega)$ is an isomorphism; see, for instance, Theorems 2.2.2.3 and 3.2.1.2 of [14] for a $C^{1,1}$ boundary $\Gamma$ and a convex domain $\Omega$, respectively. Hence, there exists a constant $C_{A}$ such that

$$
\|y\|_{H^{2}(\Omega)} \leq C_{A}\|A y\|_{L^{2}(\Omega)} \text { for all } y \in H^{2}(\Omega) .
$$

For $n=3$, we are assuming $p \geq n$, and hence $2 p /(p-2) \leq 6$. Therefore, from the Sobolev imbedding theorem, we also know that there exists a constant $M_{\Omega}$ such that

$$
\|\nabla y\|_{L^{\frac{2 p}{p-2}}(\Omega)^{n}} \leq M_{\Omega}\|y\|_{H^{2}(\Omega)} \text { for all } y \in H^{2}(\Omega) .
$$

Consider, as in the proof of Lemma 2.1, the decomposition $b=b^{\prime}+b^{\prime \prime}$, where now

$$
\left\|b^{\prime \prime}\right\|_{L^{p}(\Omega)^{n}}<\varepsilon=\frac{1}{8 C_{A} M_{\Omega}} \text { and }\left\|b^{\prime}\right\|_{L^{\infty}(\Omega)^{n}}<K_{\varepsilon, b} .
$$


Consider also two sequences $\left\{b_{k}^{\prime \prime}\right\}_{k=1}^{\infty} \subset L^{\infty}(\Omega)^{n}$ and $\left\{a_{0, k}\right\}_{k=1}^{\infty} \subset L^{\infty}(\Omega)$ such that $b_{k}^{\prime \prime} \rightarrow b^{\prime \prime}$ strongly in $L^{p}(\Omega)^{n}$ and $0 \leq a_{k} \rightarrow a_{0}$ strongly in $L^{2}(\Omega)$. Denote $b_{k}=b^{\prime}+b_{k}^{\prime \prime}$ and define the operator $\mathcal{A}_{k}: H^{2}(\Omega) \cap H_{0}^{1}(\Omega) \longrightarrow L^{2}(\Omega)$ by

$$
\mathcal{A}_{k} z=A z+b_{k}(x) \cdot \nabla z+a_{0, k}(x) z .
$$

Let us prove that $\mathcal{A}_{k}: H^{2}(\Omega) \cap H_{0}^{1}(\Omega) \rightarrow L^{2}(\Omega)$ is an isomorphism. The proof of the continuity and injectivity follows as we $\operatorname{did}$ for $\mathcal{A}$. Now, from Theorem 2.2 we deduce the existence of an element $y_{k} \in H_{0}^{1}(\Omega)$ such that $\mathcal{A}_{k} y_{k}=u$. This equation can be written as follows

$$
A y_{k}=u-b_{k}(x) \cdot \nabla y_{k}-a_{0, k}(x) y_{k}
$$

We have the estimates

$$
\begin{aligned}
& \left\|b_{k} \cdot \nabla y_{k}\right\|_{L^{2}(\Omega)} \leq\left\|b_{k}\right\|_{L^{\infty}(\Omega)^{n}}\left\|\nabla y_{k}\right\|_{L^{2}(\Omega)^{n}} \\
& \left\|a_{0, k} y_{k}\right\|_{L^{2}(\Omega)} \leq\left\|a_{0, k}\right\|_{L^{\infty}(\Omega)}\left\|y_{k}\right\|_{L^{2}(\Omega)} .
\end{aligned}
$$

Thus, we have that $A y_{k} \in L^{2}(\Omega)$. From here and (2.7) we infer

$$
\begin{aligned}
& \left\|y_{k}\right\|_{H^{2}(\Omega)} \leq C_{A}\left\|u-b_{k} \cdot \nabla y_{k}-a_{0, k} y_{k}\right\|_{L^{2}(\Omega)} \\
& \leq C_{A}\left(\|u\|_{L^{2}(\Omega)}+\left\|b^{\prime} \cdot \nabla y_{k}\right\|_{L^{2}(\Omega)}+\left\|b_{k}^{\prime \prime} \cdot \nabla y_{k}\right\|_{L^{2}(\Omega)}+\left\|a_{0, k} y_{k}\right\|_{L^{2}(\Omega)}\right) \\
& \leq C_{A}\left(\|u\|_{L^{2}(\Omega)}+\left\|b^{\prime}\right\|_{L^{\infty}(\Omega)^{n}}\left\|\nabla y_{k}\right\|_{L^{2}(\Omega)^{n}}+\left\|b_{k}^{\prime \prime}\right\|_{L^{p}(\Omega)^{n}}\left\|\nabla y_{k}\right\|_{L^{\frac{2 p}{p-2}(\Omega)^{n}}}+\left\|a_{0, k}\right\|_{L^{2}(\Omega)}\left\|y_{k}\right\|_{L^{\infty}(\Omega)}\right) .
\end{aligned}
$$

From the strong convergences of the sequences $\left\{b_{k}^{\prime \prime}\right\}_{k=1}^{\infty}$ and $\left\{a_{0, k}\right\}_{k=1}^{\infty}$, we deduce the existence of some integer $k_{0}$ such that

$$
\left\|b_{k}^{\prime \prime}\right\|_{L^{p}(\Omega)^{n}} \leq 2\left\|b^{\prime \prime}\right\|_{L^{p}(\Omega)^{n}} \text { and }\left\|a_{0, k}\right\|_{L^{2}(\Omega)} \leq 2\left\|a_{0}\right\|_{L^{2}(\Omega)} \quad \forall k \geq k_{0} .
$$

Inserting these inequalities in the above expression and taking into account the relations $\left\|b^{\prime}\right\|_{L^{\infty}(\Omega)^{n}} \leq K_{\varepsilon, b}$ and $\left\|b^{\prime \prime}\right\|_{L^{p}(\Omega)^{n}}<\varepsilon$, we infer $\forall k \geq k_{0}$

$$
\left\|y_{k}\right\|_{H^{2}(\Omega)} \leq C_{A}\left(\|u\|_{L^{2}(\Omega)}+K_{\varepsilon, b}\left\|\nabla y_{k}\right\|_{L^{2}(\Omega)^{n}}+2 \varepsilon\left\|\nabla y_{k}\right\|_{L^{\frac{2 p}{p-2}}(\Omega)^{n}}+2\left\|a_{0}\right\|_{L^{2}(\Omega)}\left\|y_{k}\right\|_{L^{\infty}(\Omega)}\right) .
$$

Now, we apply Lions' Lemma to the embeddings $H^{2}(\Omega) \subset H^{1}(\Omega) \subset L^{2}(\Omega)$ and $H^{2}(\Omega) \subset C(\bar{\Omega}) \subset L^{2}(\Omega)$, where the first embedding in each of the chains is compact. Selecting

$$
\lambda=\frac{1}{4 C_{A} \max \left\{K_{\varepsilon, b}, 2\left\|a_{0}\right\|_{L^{2}(\Omega)}\right\}},
$$

we deduce the existence of a constant $C_{\lambda}$ such that for all $y \in H^{2}(\Omega) \cap H_{0}^{1}(\Omega)$

$$
\|\nabla y\|_{L^{2}(\Omega)^{n}} \leq \lambda\|y\|_{H^{2}(\Omega)}+C_{\lambda}\|y\|_{L^{2}(\Omega)}
$$

and

$$
\|y\|_{L^{\infty}(\Omega)} \leq \lambda\|y\|_{H^{2}(\Omega)}+C_{\lambda}\|y\|_{L^{2}(\Omega)}
$$


Inserting these two inequalities in the estimate (2.9), using (2.8), and taking into account the definition of $\varepsilon$, we get

$$
\left\|y_{k}\right\|_{H^{2}(\Omega)} \leq C_{A}\left(\|u\|_{L^{2}(\Omega)}+C_{\lambda}\left[K_{\varepsilon, b}+2\left\|a_{0}\right\|_{L^{2}(\Omega)}\right]\left\|y_{k}\right\|_{L^{2}(\Omega)}\right)+\frac{3}{4}\left\|y_{k}\right\|_{H^{2}(\Omega)},
$$

which, leads to the desired estimate:

$$
\left\|y_{k}\right\|_{H^{2}(\Omega)} \leq 4 C_{A}\left(\|u\|_{L^{2}(\Omega)}+C_{\lambda}\left[K_{\varepsilon, b}+\left\|a_{0}\right\|_{L^{2}(\Omega)}\right]\left\|y_{k}\right\|_{L^{2}(\Omega)}\right)
$$

For $n=2$ the proof is slightly different. We take $\left\{b_{k}\right\}_{k=1}^{\infty} \subset L^{\infty}(\Omega)^{n}$ such that $b_{k} \rightarrow b$ strongly in $L^{p}(\Omega)^{n}$. In the same way as before we obtain that $y_{k} \in H^{2}(\Omega)$, but we directly write the estimate

$$
\begin{aligned}
& \left\|y_{k}\right\|_{H^{2}(\Omega)} \leq C_{A}\left\|u-b_{k} \cdot \nabla y_{k}-a_{0, k} y_{k}\right\|_{L^{2}(\Omega)} \\
& \leq C_{A}\left(\|u\|_{L^{2}(\Omega)}+\left\|b_{k}\right\|_{L^{p}(\Omega)^{n}}\left\|\nabla y_{k}\right\|_{L^{\frac{2 p}{p-2}}(\Omega)^{n}}+\left\|a_{0, k}\right\|_{L^{2}(\Omega)}\left\|y_{k}\right\|_{L^{\infty}(\Omega)}\right) .
\end{aligned}
$$

Now, we do not have that $\left\|b_{k}\right\|_{L^{p}(\Omega)^{n}}$ is small, but since for $n=2$ we are assuming $p>n$ we have that $H^{2}(\Omega) \subset W^{1, \frac{2 p}{p-2}}(\Omega) \subset L^{2}(\Omega)$, the first embedding being compact, and we can argue using Lions' Lemma for the term $\left\|\nabla y_{k}\right\|_{L^{\frac{2 p}{p-2}}(\Omega)^{n}}$.

Step 2. Let us prove that $y_{k} \rightarrow y=\mathcal{A}^{-1} u$ strongly in $H_{0}^{1}(\Omega)$. We have

$$
\left\|y_{k}-y\right\|_{H_{0}^{1}(\Omega)} \leq C_{\Omega}\left\|\mathcal{A}_{k}^{-1}-\mathcal{A}^{-1}\right\|_{\mathcal{L}\left(H^{-1}(\Omega), H_{0}^{1}(\Omega)\right)}\|u\|_{L^{2}(\Omega)},
$$

it is enough to show that $\left\|\mathcal{A}_{k}^{-1}-\mathcal{A}^{-1}\right\|_{\mathcal{L}\left(H^{-1}(\Omega), H_{0}^{1}(\Omega)\right)} \rightarrow 0$ when $k \rightarrow \infty$. First we prove that $\left\{\mathcal{A}_{k}^{-1}\right\}_{k=1}^{\infty}$ is bounded in $\mathcal{L}\left(H^{-1}(\Omega), H_{0}^{1}(\Omega)\right)$. It is immediate that

$$
\left\|\mathcal{A}_{k}-\mathcal{A}\right\|_{\mathcal{L}\left(H_{0}^{1}(\Omega), H^{-1}(\Omega)\right)} \leq C_{\Omega, p}\left\|b_{k}-b\right\|_{L^{\frac{2 p}{p-2}}(\Omega)^{n}}+C_{\Omega}\left\|a_{0 . k}-a_{0}\right\|_{L^{2}(\Omega)} \stackrel{k \rightarrow \infty}{\longrightarrow} 0
$$

Hence, there exists an integer $k_{1}$ such that

$$
\left\|\mathcal{A}_{k}-\mathcal{A}\right\|_{\mathcal{L}\left(H_{0}^{1}(\Omega), H^{-1}(\Omega)\right)} \leq \frac{1}{2\left\|\mathcal{A}^{-1}\right\|_{\mathcal{L}\left(H^{-1}(\Omega), H_{0}^{1}(\Omega)\right)}} \quad \forall k \geq k_{1}
$$

This implies that

$$
\left\|\mathcal{A}^{-1}\left(\mathcal{A}-\mathcal{A}_{k}\right)\right\|_{\mathcal{L}\left(H_{0}^{1}(\Omega), H_{0}^{1}(\Omega)\right)} \leq \frac{1}{2} \quad \forall k \geq k_{1} .
$$

Hence, the operator $I-\mathcal{A}^{-1}\left(\mathcal{A}-\mathcal{A}_{k}\right)$ is invertible in $H_{0}^{1}(\Omega)$ and its inverse is given by

$$
\left(I-\mathcal{A}^{-1}\left(\mathcal{A}-\mathcal{A}_{k}\right)\right)^{-1}=\sum_{j=0}^{\infty}\left[\mathcal{A}^{-1}\left(\mathcal{A}-\mathcal{A}_{k}\right)\right]^{j}
$$


From here we obtain for every $k \geq k_{1}$

$$
\left\|\left(I-\mathcal{A}^{-1}\left(\mathcal{A}-\mathcal{A}_{k}\right)\right)^{-1}\right\|_{\mathcal{L}\left(H_{0}^{1}(\Omega), H_{0}^{1}(\Omega)\right)} \leq \sum_{j=0}^{\infty}\left\|\mathcal{A}^{-1}\left(\mathcal{A}-\mathcal{A}_{k}\right)\right\|_{\mathcal{L}\left(H_{0}^{1}(\Omega), H_{0}^{1}(\Omega)\right)}^{j} \leq 2 .
$$

Now, we observe that $\mathcal{A}_{k}^{-1}=\left(I-\mathcal{A}^{-1}\left(\mathcal{A}-\mathcal{A}_{k}\right)\right)^{-1} \mathcal{A}^{-1}$. Thus, we obtain that

$$
\left\|\mathcal{A}_{k}^{-1}\right\|_{\mathcal{L}\left(H^{-1}(\Omega), H_{0}^{1}(\Omega)\right)} \leq 2\left\|\mathcal{A}^{-1}\right\|_{\mathcal{L}\left(H^{-1}(\Omega), H_{0}^{1}(\Omega)\right)} \quad \forall k \geq k_{1}
$$

Finally, we find with (2.11) and (2.12) that

$$
\begin{aligned}
& \left\|\mathcal{A}_{k}^{-1}-\mathcal{A}^{-1}\right\|_{\mathcal{L}\left(H^{-1}(\Omega), H_{0}^{1}(\Omega)\right)}=\left\|\mathcal{A}^{-1}\left[\mathcal{A}-\mathcal{A}_{k}\right] \mathcal{A}_{k}^{-1}\right\|_{\mathcal{L}\left(H^{-1}(\Omega), H_{0}^{1}(\Omega)\right)} \\
& \leq\left\|\mathcal{A}^{-1}\right\|_{\mathcal{L}\left(H^{-1}(\Omega), H_{0}^{1}(\Omega)\right)}\left\|\mathcal{A}-\mathcal{A}_{k}\right\|_{\mathcal{L}\left(H_{0}^{1}(\Omega), H^{-1}(\Omega)\right)}\left\|\mathcal{A}_{k}^{-1}\right\|_{\mathcal{L}\left(H^{-1}(\Omega), H_{0}^{1}(\Omega)\right)} \\
& \leq 2\left\|\mathcal{A}^{-1}\right\|_{\mathcal{L}\left(H^{-1}(\Omega), H_{0}^{1}(\Omega)\right)}^{2}\left\|\mathcal{A}-\mathcal{A}_{k}\right\|_{\mathcal{L}\left(H_{0}^{1}(\Omega), H^{-1}(\Omega)\right)} \stackrel{k \rightarrow \infty}{\longrightarrow} 0 .
\end{aligned}
$$

Step 3. Finally, the estimate (2.10) and the convergence $y_{k} \rightarrow y$ in $H_{0}^{1}(\Omega)$ yields $y_{k} \rightarrow y$ weakly in $H^{2}(\Omega)$. Since $u \in L^{2}(\Omega)$ was arbitrary, this implies the surjectivity of $\mathcal{A}$.

Corollary 2.6. Under the assumptions of Theorem 2.5 and in addition $\operatorname{div} b \in L^{2}(\Omega)$, the operator $\mathcal{A}^{*}: H^{2}(\Omega) \cap$ $H_{0}^{1}(\Omega) \longrightarrow L^{2}(\Omega)$ is an isomorphism.

Proof. Injectivity follows from Corollary 2.4. Let us prove that it is surjective. Take $f \in L^{2}(\Omega)$ and let $\varphi \in H_{0}^{1}(\Omega)$ be the unique solution of $\mathcal{A}^{*} \varphi=f$. Notice that, from the second part of Corollary 2.4 we also know that $\varphi \in L^{\infty}(\Omega)$. Taking into account that we can write

$$
\mathcal{A}^{*} \varphi=A^{*} \varphi-b(x) \cdot \nabla \varphi+\left(a_{0}(x)-\operatorname{div} b(x)\right) \varphi
$$

we have that

$$
A^{*} \varphi-b(x) \cdot \nabla \varphi+a_{0}(x) \varphi=f+\operatorname{div} b(x) \varphi \text { in } \Omega, \varphi=0 \text { on } \Gamma
$$

and the result follows from Theorem 2.5 because $f+\operatorname{div} b(x) \varphi \in L^{2}(\Omega)$.

\subsection{Analysis of the semilinear equation}

Here, we analyze the equation (1.1). To deal with this equation we make the following hypotheses on the nonlinear term $f$.

Assumption 2. We assume that $f: \Omega \times \mathbb{R} \longrightarrow \mathbb{R}$ is a Carathéodory function monotone nondecreasing with respect to the second variable satisfying:

$$
\forall M>0 \exists \phi_{M} \in L^{\bar{p}}(\Omega):|f(x, y)| \leq \phi_{M}(x) \text { for a.a. } x \in \Omega \text { and } \forall|y| \leq M .
$$

Let us recall that $\bar{p}$ stands for a real number bigger than $\frac{n}{2}$.

Now, we prove the existence and uniqueness of a solution for problem (1.1).

Theorem 2.7. Under the Assumptions 1 and 2, for every $u \in L^{\bar{p}}(\Omega)$ the equation (1.1) has a unique solution $y_{u}$ in $H_{0}^{1}(\Omega) \cap C(\bar{\Omega})$. Moreover, there exists a constant $K_{f}$ independent of $u$ such that

$$
\left\|y_{u}\right\|_{H_{0}^{1}(\Omega)}+\left\|y_{u}\right\|_{C(\bar{\Omega})} \leq K_{f}\left(\|u\|_{L^{\bar{p}}(\Omega)}+\|f(\cdot, 0)\|_{L^{\bar{p}}(\Omega)}+1\right) .
$$


Before proving this theorem we establish the following lemma.

Lemma 2.8. Let $g: \Omega \times \mathbb{R} \longrightarrow \mathbb{R}$ be a function satisfying Assumption 2. We also suppose that Assumption 1 holds. Then, if $y_{1}, y_{2} \in H_{0}^{1}(\Omega) \cap L^{\infty}(\Omega)$ are solutions of the equations

$$
A y_{i}+b(x) \cdot \nabla y_{i}+g\left(x, y_{i}\right)=u_{i}, \quad i=1,2,
$$

with $u_{1}, u_{2} \in L^{\bar{p}}(\Omega)$ and $u_{1} \leq u_{2}$ in $\Omega$, then $y_{1} \leq y_{2}$ in $\Omega$ as well.

Proof. We make the proof for $n=3$. The case $n=2$ can be proved in a similar way. We argue by contradiction, proceeding similarly to the proof of Theorem 2.2. If the statement of the lemma is false, then there exists $0<\rho<\operatorname{esssup}_{x \in \Omega}\left(y_{1}(x)-y_{2}(x)\right)$. Now, we set $z(x)=\left[\left(y_{1}(x)-y_{2}(x)\right)-\rho\right]^{+}$. We have that $z \in H_{0}^{1}(\Omega)$. We denote $\Omega_{\rho}=\{x \in \Omega: \nabla z(x) \neq 0\}$. Let us observe that we have

$$
\nabla z(x)=\left\{\begin{array}{cl}
\nabla\left(y_{1}-y_{2}\right)(x) & \text { if }\left(y_{1}-y_{2}\right)(x)>\rho \\
0 & \text { otherwise }
\end{array}\right.
$$

and

$$
z(x)=0 \text { if }\left(y_{1}-y_{2}\right)(x) \leq \rho .
$$

Using these facts, our assumptions on $b$ and $u_{i}$, and the monotonicity of $g$ we get

$$
\begin{aligned}
0 \geq & \int_{\Omega}\left(u_{1}-u_{2}\right) z \mathrm{~d} x \\
= & \int_{\Omega}\left(\sum_{i, j=1}^{n} a_{i j}(x) \partial_{x_{i}}\left(y_{1}-y_{2}\right) \partial_{x_{j}} z+\left[b(x) \cdot \nabla\left(y_{1}-y_{2}\right)\right] z\right) \mathrm{d} x \\
& +\int_{\Omega}\left[g\left(x, y_{1}\right)-g\left(x, y_{2}\right)\right] z \mathrm{~d} x \geq \int_{\Omega_{\rho}}\left(\sum_{i, j=1}^{n} a_{i j}(x) \partial_{x_{i}} z \partial_{x_{j}} z+[b(x) \cdot \nabla z] z\right) \mathrm{d} x \\
\geq & \Lambda\|\nabla z\|_{L^{2}\left(\Omega_{\rho}\right)^{n}}^{2}-\|b\|_{L^{3}\left(\Omega_{\rho}\right)^{n}}\|\nabla z\|_{L^{2}\left(\Omega_{\rho}\right)^{n}}\|z\|_{L^{6}\left(\Omega_{\rho}\right)} .
\end{aligned}
$$

Now, we continue as in the proof of Theorem 2.2 to achieve the contradiction

$$
\|b\|_{L^{3}\left(\Omega_{\rho}\right)^{n}} \geq \frac{\Lambda}{C_{1}}>0 \quad \forall \rho<\operatorname{ess} \sup _{x \in \Omega}\left(y_{1}-y_{2}\right)(x) .
$$

Proof of Theorem 2.7. The uniqueness of a solution is an immediate consequence of Lemma 2.8. The proof of existence is divided into three steps according to different assumptions on $f$. To simplify the presentation, we redefine $f=f-f(\cdot, 0)$ and $u=u-f(\cdot, 0) \in L^{\bar{p}}(\Omega)$. Then, due to Assumption $2, f$ is continuous and monotone nondecreasing, $f(x, 0)=0$, and $f$ is dominated by a function $\phi_{M} \in L^{\bar{p}}(\Omega)$ in $\Omega \times[-M,+M]$ for every $M>0$.

Step 1. Assume that there exists $\phi \in L^{\bar{p}}(\Omega)$ such that $|f(x, y)| \leq \phi(x)$ in $\Omega \times \mathbb{R}$. In this case, we consider the operator $T: C(\bar{\Omega}) \longrightarrow C(\bar{\Omega})$ given by $T w=y_{w}$, where $y_{w}$ is the solution of the problem

$$
\left\{\begin{array}{l}
A y+b(x) \cdot \nabla y+f(x, w)=u \text { in } \Omega \\
y=0 \text { on } \Gamma
\end{array}\right.
$$


From Corollary 2.3 we have the existence and uniqueness of a solution $y_{w} \in H_{0}^{1}(\Omega) \cap C^{0, \mu}(\bar{\Omega})$ for some $\mu \in(0,1)$. This solution satisfies

$$
\left\|y_{w}\right\|_{C^{0, \mu}(\bar{\Omega})} \leq C_{A, b}\|u-f(x, w)\|_{L^{\bar{p}}(\Omega)} \leq C_{A, b}\left(\|u\|_{L^{\bar{p}}(\Omega)}+\|\phi\|_{L^{\bar{p}}(\Omega)}\right)=\rho .
$$

From the compactness of the embedding $C^{0, \mu}(\bar{\Omega}) \subset C(\bar{\Omega})$, we deduce that $T$ is a compact operator applying the closed ball $\bar{B}_{\rho}(0)$ into itself. Hence, from Schauder's fixed point Theorem we infer the existence of a solution $y_{u} \in H_{0}^{1}(\Omega) \cap C(\bar{\Omega})$ of (1.1). Moreover, (2.14) follows from the above inequality and the redefinition of $u$ along with the fact that $A+b \cdot \nabla I: H_{0}^{1}(\Omega) \longrightarrow H^{-1}(\Omega)$ is an isomorphism.

Step 2. We relax the assumption of step 1 and now we only assume that there exists $\phi \in L^{\bar{p}}(\Omega)$ such that $f \geq \phi$ in $\Omega \times \mathbb{R}$. For every integer $k \geq 1$ we set $f_{k}(x, y)=f(x, \min \{y, k\})$. Then, from (2.13) we infer

$$
\phi(x) \leq f_{k}(x, y) \leq f\left(x, \operatorname{proj}_{[-k,+k]}(y)\right) \leq \phi_{k}(x) .
$$

Hence, $\left|f_{k}(x, y)\right| \leq \psi_{k}(x)=\max \left\{|\phi(x)|,\left|\phi_{k}(x)\right|\right\}$ with $\psi_{k} \in L^{\bar{p}}(\Omega)$. Then, we can apply Step 1 to deduce the existence of a function $y_{k} \in H_{0}^{1}(\Omega) \cap C(\bar{\Omega})$ satisfying

$$
\left\{\begin{array}{l}
A y_{k}+b(x) \cdot \nabla y_{k}+f_{k}\left(x, y_{k}\right)=u \text { in } \Omega \\
y_{k}=0 \text { on } \Gamma
\end{array}\right.
$$

Now, by Corollary 2.3 there exists a function $y \in H_{0}^{1}(\Omega) \cap C(\bar{\Omega})$ solution of

$$
\left\{\begin{array}{l}
A y+b(x) \cdot \nabla y=u-\phi \text { in } \Omega \\
y=0 \text { on } \Gamma
\end{array}\right.
$$

Subtracting both equations we get

$$
A\left(y_{k}-y\right)+b(x) \cdot \nabla\left(y_{k}-y\right)=-f_{k}\left(x, y_{k}\right)+\phi \leq 0 .
$$

Then, Lemma 2.8 implies that $y_{k} \leq y$ in $\Omega$. Therefore, if we take $k>\|y\|_{C(\bar{\Omega})}$, we have that $f_{k}\left(x, y_{k}\right)=f\left(x, y_{k}\right)$, and therefore $y_{k}$ is solution of (1.1). The estimate (2.14) follows from the bound for $y_{k}$ independently of $k$ and Assumption 2.

Step 3. The general case. Let us define $f_{k}(x, y)=f\left(x, \operatorname{proj}_{[-k,+k]}(y)\right)$. Then, according to Assumption 2, there exists a function $\phi_{k} \in L^{\bar{p}}(\Omega)$ such that $\left|f_{k}(x, y)\right| \leq \phi_{k}(x)$ in $\Omega \times \mathbb{R}$. Therefore, from Step 1 we know that there exists $y_{k} \in H_{0}^{1}(\Omega) \cap C(\bar{\Omega})$ satisfying

$$
A y_{k}+b(x) \cdot \nabla y_{k}+f_{k}\left(x, y_{k}\right)=u \text {. }
$$

Now, we take $z_{1} \in H_{0}^{1}(\Omega) \cap C(\bar{\Omega})$ satisfying $A z_{1}+b(x) \cdot \nabla z_{1}+f\left(x, z_{1}^{+}\right)=u$. The existence of such a function follows from Step 2 because $f\left(x, z_{1}^{+}\right) \geq 0$. This equation can be written as

$$
A z_{1}+b(x) \cdot \nabla z_{1}+f_{k}\left(x, z_{1}\right)=u+f_{k}\left(x, z_{1}\right)-f\left(x, z_{1}^{+}\right) .
$$

From the monotonicity of $f$ with respect to the second variable, we have that $f_{k}\left(x, z_{1}\right)-f\left(x, z_{1}^{+}\right) \leq 0$, and hence Lemma 2.8 implies that $z_{1} \leq y_{k}$ in $\Omega$.

Now, let $z_{2} \in H_{0}^{1}(\Omega) \cap C(\bar{\Omega})$ satisfy

$$
A z_{2}+b(x) \cdot \nabla z_{2}=u-f\left(x,-\left\|z_{1}\right\|_{C(\bar{\Omega})}\right)
$$


The existence of such a function follows from Theorem 2.2 and Corollary 2.3. Indeed, it is enough to observe that from (2.13) with $M=\left\|z_{1}\right\|_{C(\bar{\Omega})}$ we deduce the existence of a function $\phi_{M} \in L^{\bar{p}}(\Omega)$ such that $\left|f\left(x,-\left\|z_{1}\right\|_{C(\bar{\Omega})}\right)\right| \leq$ $\phi_{M}(x)$ for almost all $x \in \Omega$. Then, $f\left(\cdot,-\left\|z_{1}\right\|_{C(\bar{\Omega})}\right) \in L^{\bar{p}}(\Omega)$ holds. Noticing that the equation satisfied by $y_{k}$ can be written

$$
A y_{k}+b(x) \cdot \nabla y_{k}=u-f_{k}\left(x, y_{k}\right)
$$

and that, using the fact that $y_{k} \geq z_{1} \geq-\left\|z_{1}\right\|_{C(\bar{\Omega})}$, we have $f_{k}\left(x, y_{k}(x)\right) \geq f\left(x,-\left\|z_{1}\right\|_{C(\bar{\Omega})}\right)$. Then, subtracting the equations satisfied by $y_{k}$ and $z_{2}$ we get

$$
A\left(z_{2}-y_{k}\right)+b(x) \cdot \nabla\left(z_{2}-y_{k}\right)=f_{k}\left(x, y_{k}\right)-f\left(x,-\left\|z_{1}\right\|_{C(\bar{\Omega})}\right) \geq 0 .
$$

Therefore, $z_{1} \leq y_{k} \leq z_{2}$ and, hence, $f_{k}\left(x, y_{k}\right)=f\left(x, y_{k}\right)$ for every $k>\max \left\{\left\|z_{1}\right\|_{C(\bar{\Omega})},\left\|z_{2}\right\|_{C(\bar{\Omega})}\right\}$, and consequently $y_{k}$ is solution of (1.1) for $k$ large enough. As in the previous step, the estimate (2.14) follows from the bound for $y_{k}$ independently of $k$.

Now, we establish some additional regularity for the solutions of (1.1).

Corollary 2.9. There exists $\mu \in(0,1)$ such that the solution $y_{u}$ of $(1.1)$ belongs to $C^{0, \mu}(\bar{\Omega})$. Moreover, for every $M>0$ there exists a constant $K_{f, \mu, M}$ such that

$$
\left\|y_{u}\right\|_{C^{0, \mu}(\bar{\Omega})} \leq K_{f, \mu, M} \quad \forall u \in L^{\bar{p}}(\Omega) \text { satisfying }\|u\|_{L^{\bar{p}}(\Omega)} \leq M .
$$

Proof. Since $A y_{u}+b \cdot \nabla y_{u}=u-f\left(x, y_{u}\right)$, this corollary follows from (2.13), (2.14) and Corollary 2.3.

Theorem 2.10. Suppose that Assumption 1 holds, $a_{i j} \in C^{0,1}(\bar{\Omega})$ for $1 \leq i, j \leq n$ and $a_{0} \in L^{2}(\Omega)$. We also suppose that Assumption 2 holds with $\bar{p}=2$, and that $\Gamma$ is of class $C^{1,1}$ or $\Omega$ is convex. Then, for every $u \in L^{2}(\Omega)$, the equation (1.1) has a unique solution $y_{u} \in H^{2}(\Omega) \cap H_{0}^{1}(\Omega)$. Moreover, for every $M>0$ there exists a constant $C_{\mathcal{A}, f, M}$ such that

$$
\left\|y_{u}\right\|_{H^{2}(\Omega)} \leq C_{\mathcal{A}, f, M} \quad \forall u \in L^{\bar{p}}(\Omega) \text { satisfying }\|u\|_{L^{\bar{p}}(\Omega)} \leq M
$$

This is an immediate consequence of Theorems 2.5 and 2.7. The following result on the continuous dependence of the state $y_{u}$ respect to $u$ will be useful to prove the existence of a solution for the control problem (P).

Theorem 2.11. Let $\left\{u_{k}\right\}_{k=1}^{\infty} \subset L^{\bar{p}}(\Omega)$ be a sequence weakly converging to $u$ in $L^{\bar{p}}(\Omega)$. Then, under the assumptions of Theorem 2.7 we have that $y_{u_{k}} \rightarrow y_{u}$ strongly in $H_{0}^{1}(\Omega) \cap C(\bar{\Omega})$.

Proof. From Theorem 2.7 and Corollary 2.9 we know that $\left\{y_{u_{k}}\right\}_{k=1}^{\infty}$ is bounded in $H_{0}^{1}(\Omega) \cap C^{0, \mu}(\bar{\Omega})$. Hence, using the compactness of the embedding $C^{0, \mu}(\bar{\Omega}) \subset C(\bar{\Omega})$, we deduce the existence of a subsequence, denoted in the same way, and an element $y \in H_{0}^{1}(\Omega) \cap C(\bar{\Omega})$ such that $y_{u_{k}} \rightarrow y$ in $H_{0}^{1}(\Omega)$ and $y_{u_{k}} \rightarrow y$ in $C(\bar{\Omega})$. Now, setting $M=\max _{1 \leq k<\infty}\left\|y_{u_{k}}\right\|_{C(\bar{\Omega})}$, we deduce from (2.13)

$$
\begin{gathered}
\left|f\left(x, y_{u_{k}}(x)\right)\right| \leq|f(x, 0)|+\left|f\left(x, y_{u_{k}}(x)\right)-f(x, 0)\right| \\
\leq|f(x, 0)|+C_{f, M}\left|y_{u_{k}}(x)\right| \leq|f(x, 0)|+C_{f, M} M .
\end{gathered}
$$

As a consequence we have that $f\left(x, y_{u_{k}}\right) \rightarrow f(x, y)$ strongly in $L^{\bar{p}}(\Omega)$. Moreover, the compactness of the embed$\operatorname{ding} L^{\bar{p}}(\Omega) \subset H^{-1}(\Omega)$ implies that $u_{k} \rightarrow u$ strongly in $H^{-1}(\Omega)$. Hence, from Theorem 2.2 we infer that $y_{u_{k}}=(A+b \cdot \nabla I)^{-1}\left(u_{k}-f\left(x, y_{u_{k}}\right)\right) \rightarrow(A+b \cdot \nabla I)^{-1}(u-f(x, y))$ strongly in $H_{0}^{1}(\Omega)$. Thus, we have that 
$y=y_{u}$ and $y_{u_{k}} \rightarrow y_{u}$ strongly in $H_{0}^{1}(\Omega) \cap C(\bar{\Omega})$. Since, this convergence holds for any converging subsequence, we deduce that the whole sequence converges as indicated in the statement of the theorem.

To finish this section we analyze the differentiability of the relation $u \rightarrow y_{u}$. To this end, we make the following assumptions on $f$.

Assumption 3. We assume that $f: \Omega \times \mathbb{R} \longrightarrow \mathbb{R}$ is a Carathéodory function of class $C^{2}$ with respect to the second variable satisfying:

$$
f(\cdot, 0) \in L^{\bar{p}}(\Omega) \text { and } \frac{\partial f}{\partial y}(x, y) \geq 0 \text { a.e. in } \Omega \text { and } \forall y \in \mathbb{R},
$$

and for all $M>0$ there exists a constant $C_{f, M}>0$ such that

$$
\left|\frac{\partial f}{\partial y}(x, y)\right|+\left|\frac{\partial^{2} f}{\partial y^{2}}(x, y)\right| \leq C_{f, M} \text { for a.e. } x \in \Omega \text { and for all }|y| \leq M \text {. }
$$

For every $M>0$ and $\varepsilon>0$ there exists $\delta>0$, depending on $M$ and $\varepsilon$, such that

$$
\left|\frac{\partial^{2} f}{\partial y^{2}}\left(x, y_{2}\right)-\frac{\partial^{2} f}{\partial y^{2}}\left(x, y_{1}\right)\right|<\varepsilon \text { if }\left|y_{1}\right|,\left|y_{2}\right| \leq M,\left|y_{2}-y_{1}\right| \leq \delta \text {, for a.a. } x \in \Omega \text {. }
$$

Let us recall again that $\bar{p}$ stands for a real number bigger than $\frac{n}{2}$. It is obvious that Assumption 3 implies Assumption 2. Therefore, all the previous results remain valid if we replace Assumption 2 by Assumption 3.

Theorem 2.12. Let us suppose that Assumptions 1 and 3 hold. Then, the mapping $G: L^{\bar{p}}(\Omega) \longrightarrow H_{0}^{1}(\Omega) \cap C(\bar{\Omega})$ given by $G(u)=y_{u}$ is well defined and of class $C^{2}$. Moreover, given $u, v \in L^{\bar{p}}(\Omega), z_{v}=D G(u) v$ is the solution of

$$
\left\{\begin{array}{l}
A z+b(x) \cdot \nabla z+\frac{\partial f}{\partial y}\left(x, y_{u}\right) z=v \text { in } \Omega \\
z=0 \text { on } \Gamma
\end{array}\right.
$$

For $v_{1}, v_{2} \in L^{\bar{p}}(\Omega)$ the second derivative $z_{v_{1}, v_{2}}=D^{2} G(u)\left(v_{1}, v_{2}\right)$ is the solution of the equation

$$
\left\{\begin{array}{l}
A z+b(x) \cdot \nabla z+\frac{\partial f}{\partial y}\left(x, y_{u}\right) z=-\frac{\partial^{2} f}{\partial y^{2}}\left(x, y_{u}\right) z_{v_{1}} z_{v_{2}} \text { in } \Omega \\
z=0 \text { on } \Gamma
\end{array}\right.
$$

where $z_{v_{i}}=D G(u) v_{i}, i=1,2$.

Proof. The fact that $G$ is well defined is a straightforward consequence of Theorem 2.7. To prove the differentiability we will use the implicit function theorem as follows. We consider the vector space

$$
Y=\left\{y \in H_{0}^{1}(\Omega) \cap C(\bar{\Omega}): A y+b \cdot \nabla y \in L^{\bar{p}}(\Omega)\right\} .
$$

This is a Banach space when we endow it with the norm

$$
\|y\|_{Y}=\|y\|_{H_{0}^{1}(\Omega)}+\|y\|_{C(\bar{\Omega})}+\|A y+b \cdot \nabla y\|_{L^{\bar{p}}(\Omega)} .
$$

Let us consider the mapping

$$
\mathcal{F}: Y \times L^{\bar{p}}(\Omega) \longrightarrow L^{\bar{p}}(\Omega)
$$


given by

$$
\mathcal{F}(y, u)=A y+b(x) \cdot \nabla y+f(\cdot, y)-u
$$

Using Assumption 3 and Corollary 2.3 it is easy to check that $f(\cdot, y) \in L^{\bar{p}}(\Omega)$ for every $y \in Y$, and the mapping $y \in Y \rightarrow f(\cdot, y) \in L^{\bar{p}}(\Omega)$ is of class $C^{2}$. Hence, $\mathcal{F}$ is well defined and it is of class $C^{2}$. Moreover, the linear mapping

$$
\begin{gathered}
\frac{\partial \mathcal{F}}{\partial y}(y, u): Y \longrightarrow L^{\bar{p}}(\Omega) \\
\frac{\partial \mathcal{F}}{\partial y}(y, u) z=A z+b(x) \cdot \nabla z+\frac{\partial f}{\partial y}(x, y) z
\end{gathered}
$$

is an isomorphism. Indeed, if we consider the operator $\mathcal{A}$ defined by $(2.2)$ with $a_{0}(x)=\frac{\partial f}{\partial y}(x, y(x))$, we have to prove that $\mathcal{A}: Y \longrightarrow L^{\bar{p}}(\Omega)$ is an isomorphism. From the definition of $Y$ and the above estimates, we know that $\mathcal{A}$ is well defined and continuous. From Theorem 2.2 we also deduce the existence of a unique solution $z \in H_{0}^{1}(\Omega)$ of the equation $\mathcal{A} z=v$ for every $v \in L^{\bar{p}}(\Omega) \subset H^{-1}(\Omega)$. In addition, from Corollary 2.3 we know that $z \in C(\bar{\Omega})$. Hence, we have that $z \in Y$ and $\mathcal{A}$ is an isomorphism. Then, we can apply the implicit function theorem and deduce easily the theorem; see e.g. Proposition 16 in [6].

\section{Analysis of the optimal CONTRol PRoblem}

In this section, we firstly prove the existence of a global solution $\bar{u}$ of the control problem. Then, we derive first and second order necessary optimality conditions for local solutions. Finally, we prove sufficient conditions for local optimality. In the whole section we suppose that Assumptions 1 and 3 are fulfilled.

Theorem 3.1. Let us assume that $L: \Omega \times \mathbb{R} \longrightarrow \mathbb{R}$ is a Carathédory function satisfying

$$
\forall M>0 \exists \psi_{M} \in L^{1}(\Omega):|L(x, y)| \leq \psi_{M}(x) \text { for a.a. } x \in \Omega \text { and } \forall|y| \leq M .
$$

Then, if $U_{\mathrm{ad}}$ is bounded in $L^{2}(\Omega)$ or $L$ is bounded from below, the control problem $(\mathrm{P})$ has at least one solution $\bar{u}$.

Proof. Let $\left\{u_{k}\right\}_{k=1}^{\infty} \subset U_{\text {ad }}$ be a minimizing sequence of (P). From the boundedness of $U_{\text {ad }}$ or the lower boundedness of $L$ we deduce that $\left\{u_{k}\right\}_{k=1}^{\infty}$ is bounded in $L^{2}(\Omega)$. Hence, we can take a subsequence, denoted in the same way, converging weakly in $L^{2}(\Omega)$ to some element $\bar{u}$. Since $U_{\text {ad }}$ is weakly closed in $L^{2}(\Omega)$ we infer that $\bar{u} \in U_{\text {ad }}$. Moreover, Theorem 2.5 implies that $y_{u_{k}} \rightarrow y_{\bar{u}}$ strongly in $H_{0}^{1}(\Omega) \cap C(\bar{\Omega})$. Therefore, using the assumption (3.1) along with Lebesgue's dominated convergence theorem, we get that $J(\bar{u}) \leq \liminf _{k \rightarrow \infty} J\left(u_{k}\right)=\inf (\mathrm{P})$ and, hence, $\bar{u}$ is a solution of $(\mathrm{P})$.

Before establishing the optimality conditions for $(\mathrm{P})$, we study the differentiability of $J$. To this end we make the following assumptions on $L$.

Assumption 4. We assume that $L: \Omega \times \mathbb{R} \longrightarrow \mathbb{R}$ is a Carathéodory function of class $C^{2}$ with respect to the second variable satisfying that $L(\cdot, 0) \in L^{1}(\Omega)$ and for all $M>0$ there exist a function $\psi_{M} \in L^{\bar{p}}(\Omega)$ with $\bar{p}>\frac{n}{2}$ and a constant $C_{L, M}>0$ such that

$$
\left|\frac{\partial L}{\partial y}(x, y)\right| \leq \psi_{M}(x) \text { and }\left|\frac{\partial^{2} L}{\partial y^{2}}(x, y)\right| \leq C_{L, M} \text { for a.e. } x \in \Omega \text { and for all }|y| \leq M \text {. }
$$


In addition, for every $M>0$ and $\varepsilon>0$ there exists $\delta>0$, depending on $M$ and $\varepsilon$, such that

$$
\left|\frac{\partial^{2} L}{\partial y^{2}}\left(x, y_{2}\right)-\frac{\partial^{2} L}{\partial y^{2}}\left(x, y_{1}\right)\right|<\varepsilon \text { if }\left|y_{1}\right|,\left|y_{2}\right| \leq M,\left|y_{2}-y_{1}\right| \leq \delta, \text { for a.a. } x \in \Omega
$$

It is obvious that (3.1) holds under Assumption 4. In the rest of the paper, we will suppose that Assumptions 1, 2 and 4 are fulfilled. Then, we have the following differentiability result.

Theorem 3.2. The functional $J$ is of class $C^{2}$. Moreover, given $u, v, v_{1}, v_{2} \in L^{2}(\Omega)$ we have

$$
\begin{aligned}
& J^{\prime}(u) v=\int_{\Omega}\left(\varphi_{u}+\nu u\right) v \mathrm{~d} x, \\
& J^{\prime \prime}(u)\left(v_{1}, v_{2}\right)=\int_{\Omega}\left[\frac{\partial^{2} L}{\partial y^{2}}\left(x, y_{u}\right)-\varphi_{u} \frac{\partial^{2} f}{\partial y^{2}}\left(x, y_{u}\right)\right] z_{v_{1}} z_{v_{2}} \mathrm{~d} x+\nu \int_{\Omega} v_{1} v_{2} \mathrm{~d} x,
\end{aligned}
$$

where $\varphi_{u} \in H_{0}^{1}(\Omega) \cap C(\bar{\Omega})$ is the unique solution of the adjoint equation

$$
\left\{\begin{array}{l}
A^{*} \varphi-\operatorname{div}[b(x) \varphi]+\frac{\partial f}{\partial y}\left(x, y_{u}\right) \varphi=\frac{\partial L}{\partial y}\left(x, y_{u}\right) \text { in } \Omega \\
\varphi=0 \text { on } \Gamma
\end{array}\right.
$$

Proof. The $C^{2}$ differentiability of $J$ is an immediate consequence of Theorem 2.12, Assumption 4 and the chain rule. Moreover, the derivation of the formulas (3.4) and (3.5) is standard. The existence of a unique $\varphi_{u} \in H_{0}^{1}(\Omega) \cap C(\bar{\Omega})$ follows from Corollary 2.4 and the facts that (2.17) along with $y_{u} \in C(\bar{\Omega})$ implies that $a_{0}=\frac{\partial f}{\partial y}\left(\cdot, y_{u}\right) \in L^{\infty}(\Omega)$ and assumption $(3.2)$ implies that $\frac{\partial L}{\partial y}\left(\cdot, y_{u}\right) \in L^{\bar{p}}(\Omega)$.

Since $(\mathrm{P})$ is not a convex problem, we consider local solutions of $(\mathrm{P})$ as well. Let us state precisely the different concepts of local solution.

Definition 3.3. We say that $\bar{u} \in U_{\text {ad }}$ is an $L^{r}(\Omega)$-weak local minimum of $(\mathrm{P})$ with $r \in[1,+\infty]$, if there exists some $\varepsilon>0$ such that

$$
J(\bar{u}) \leq J(u) \quad \forall u \in U_{\text {ad }} \text { with }\|\bar{u}-u\|_{L^{r}(\Omega)} \leq \varepsilon .
$$

An element $\bar{u} \in U_{\text {ad }}$ is said a strong local minimum of (P) if there exists some $\varepsilon>0$ such that

$$
J(\bar{u}) \leq J(u) \quad \forall u \in U_{\text {ad }} \text { with }\left\|y_{\bar{u}}-y_{u}\right\|_{L^{\infty}(\Omega)} \leq \varepsilon .
$$

We say that $\bar{u} \in U_{\text {ad }}$ is a strict (weak or strong) local minimum if the above inequalities are strict for $u \neq \bar{u}$.

As far as we know, the notion of strong local solutions in the framework of control theory was introduced in [3] for the first time; see also [2]. We analyze the relationships among these concepts in the followin lemma.

Lemma 3.4. The following properties hold:

If $U_{\mathrm{ad}}$ is bounded in $L^{2}(\Omega)$, then

1. $\bar{u}$ is an $L^{1}(\Omega)$-weak local minimum of $(\mathrm{P})$ if and only if it is an $L^{r}(\Omega)$-weak local minimum of $(\mathrm{P})$ for every $r \in(1,+\infty)$.

2. If $\bar{u}$ is an $L^{r}(\Omega)$-weak local minimum of $(\mathrm{P})$ for some $r<+\infty$, then it is an $L^{\infty}(\Omega)$-weak local minimum of $(\mathrm{P})$. 
3. $\bar{u}$ is a strong local minimum of $(\mathrm{P})$ if and only if it is an $L^{r}(\Omega)$-weak local minimum of $(\mathrm{P})$ for all $r \in[1, \infty)$.

If $U_{\mathrm{ad}}$ is not bounded in $L^{2}(\Omega)$, then

1. If $\bar{u}$ is an $L^{2}(\Omega)$-weak local solution and $L$ is bounded from below, then $\bar{u}$ is an $L^{1}(\Omega)$-weak local solution.

2. If $\bar{u}$ is an $L^{p}(\Omega)$-weak local solution, then $\bar{u}$ is an $L^{q}(\Omega)$-weak local solution for every $p<q \leq \infty$.

3. $\bar{u}$ is an $L^{2}(\Omega)$-weak local solution if and only if it is a strong local solution.

The reader is referred to [7] and [11] for the proof of this lemma. To deduce that any strong local solution is an $L^{2}(\Omega)$-weak local solution the following estimate is used

$$
\left\|y_{u}-y_{\bar{u}}\right\|_{C(\bar{\Omega})} \leq C\|u-\bar{u}\|_{L^{2}(\Omega)} \quad \forall u \in B_{r}(\bar{u})
$$

where $B_{r}(\bar{u})$ is the ball in $L^{2}(\Omega)$. This inequality follows from the next result.

Lemma 3.5. Let $\mathcal{U}$ be a bounded subset of $L^{\bar{p}}(\Omega)$. Then, there exists a constant $M_{\mathcal{U}}$ such that

$$
\left\|y_{u}-y_{v}\right\|_{H_{0}^{1}(\Omega)}+\left\|y_{u}-y_{v}\right\|_{C(\bar{\Omega})} \leq M_{\mathcal{U}}\|u-v\|_{L^{\bar{p}}(\Omega)} \quad \forall u \in \mathcal{U}
$$

Proof. Without loss of generality, we can suppose that $\mathcal{U}$ is convex. Otherwise, we replace it by its convex hull, which is also a bounded set. Given $u, v \in \mathcal{U}$, from Theorem 2.12 and the mean value theorem we have

$$
\left\|y_{u}-y_{v}\right\|_{H_{0}^{1}(\Omega)}+\left\|y_{u}-y_{v}\right\|_{C(\bar{\Omega})} \leq \sup _{\hat{u} \in \mathcal{U}}\|D G(\hat{u})\|_{\mathcal{L}\left(L^{\bar{p}}(\Omega), H_{0}^{1}(\Omega) \cap C(\bar{\Omega})\right)}\|u-v\|_{L^{\bar{p}}(\Omega)} .
$$

Then, it is enough to prove that $\|D G(\hat{u})\|_{\mathcal{L}\left(L^{\bar{p}}(\Omega), H_{0}^{1}(\Omega) \cap C(\bar{\Omega})\right)}$ is bounded by a constant $M_{\mathcal{U}}$ for every $\hat{u} \in \mathcal{U}$. From Corollary 2.9, we know that $M=\sup \left\{\left\|y_{u}\right\|_{C(\bar{\Omega})}: u \in \mathcal{U}\right\}<+\infty$. Hence, from Assumption 3 we have

$$
\left|\frac{\partial f}{\partial y}\left(x, y_{u}(x)\right)\right| \leq C_{f, M} \text { for a.a. } x \in \Omega \text { and } \forall u \in \mathcal{U} \text {. }
$$

Now, given $u \in \mathcal{U}$ arbitrary and $v \in L^{\bar{p}}(\Omega)$ with $\|v\|_{L^{\bar{p}}(\Omega)}=1$, we denote by $z$ and $z_{0}$ the elements of $H_{0}^{1}(\Omega) \cap$ $C(\bar{\Omega})$ satisfying the equations

$$
\begin{aligned}
& \left\{\begin{array}{l}
A z+b(x) \cdot \nabla z+\frac{\partial f}{\partial y}\left(x, y_{u}\right) z=v \text { in } \Omega, \\
z=0 \text { on } \Gamma .
\end{array}\right. \\
& \left\{\begin{array}{l}
A z_{0}+b(x) \cdot \nabla z_{0}=|v| \text { in } \Omega, \\
z=0 \text { on } \Gamma .
\end{array}\right.
\end{aligned}
$$

Taking in Lemma $2.8 \mathrm{~g}=0, u_{1}=0$ and $u_{2}=|v|$, we deduce that $z_{0} \geq 0$. Now, subtracting and adding the equations satisfied by $z$ and $z_{0}$, and using the monotonicity of $f$ we get

$$
\begin{aligned}
& A\left(z_{0}-z\right)+b(x) \cdot \nabla\left(z_{0}-z\right)+\frac{\partial f}{\partial y}\left(x, y_{u}\right)\left(z_{0}-z\right)=\frac{\partial f}{\partial y}\left(x, y_{u}\right) z_{0}+|v|-v \geq 0 \text { in } \Omega \\
& A\left(z_{0}+z\right)+b(x) \cdot \nabla\left(z_{0}+z\right)+\frac{\partial f}{\partial y}\left(x, y_{u}\right)\left(z_{0}+z\right)=\frac{\partial f}{\partial y}\left(x, y_{u}\right) z_{0}+|v|+v \geq 0 \text { in } \Omega
\end{aligned}
$$


Using again Lemma 2.8 we infer that $z_{0}-z \geq 0$ and $z_{0}+z \geq 0$ in $\Omega$, or equivalently $-z_{0} \leq z \leq z_{0}$ in $\Omega$. Thus, we get with $(2.4)$

$$
\|z\|_{C(\bar{\Omega})} \leq\left\|z_{0}\right\|_{C(\bar{\Omega})} \leq C_{A, b}\|v\|_{L^{p}(\Omega)}=C_{A, b}
$$

On the other hand, from Theorem 2.2 we deduce the existence of a constant $C$ independent of $v$ such that

$$
\begin{aligned}
& \|z\|_{H_{0}^{1}(\Omega)} \leq C\left(\|v\|_{L^{p}(\Omega)}+\left\|\frac{\partial f}{\partial y}\left(x, y_{u}\right) z\right\|_{L^{p}(\Omega)}\right) \\
& \leq C\left(1+\left\|\frac{\partial f}{\partial y}\left(x, y_{u}\right)\right\|_{L^{\infty}(\Omega)}\|z\|_{C(\bar{\Omega})}|\Omega|^{\frac{1}{p}}\right) \leq C\left(1+C_{f, M} C_{A, b}|\Omega|^{\frac{1}{p}}\right) .
\end{aligned}
$$

Hence, we have

$$
\|z\|_{H_{0}^{1}(\Omega)}+\|z\|_{C(\bar{\Omega})} \leq C\left(1+C_{f, M} C_{A, b}|\Omega|^{\frac{1}{p}}\right)+C_{A, b}=M_{\mathcal{U}} .
$$

Since $u$ and $v$ are arbitrary, we conclude that $\|D G(\hat{u})\|_{\mathcal{L}\left(L^{\bar{p}}(\Omega), H_{0}^{1}(\Omega) \cap C(\bar{\Omega})\right)} \leq M_{\mathcal{U}}$, and the lemma follows.

Now, we establish the first order optimality conditions.

Theorem 3.6. Let $\bar{u}$ be a local solution of $(\mathrm{P})$ in any of the previous senses. Then there exist two unique elements $\bar{y}, \bar{\varphi} \in H_{0}^{1}(\Omega) \cap C(\bar{\Omega})$ such that

$$
\begin{aligned}
& \left\{\begin{array}{l}
A \bar{y}+b(x) \cdot \nabla \bar{y}+f(x, \bar{y})=\bar{u} \text { in } \Omega, \\
\bar{y}=0 \text { on } \Gamma,
\end{array}\right. \\
& \left\{\begin{array}{l}
A^{*} \bar{\varphi}-\operatorname{div}[b(x) \bar{\varphi}]+\frac{\partial f}{\partial y}(x, \bar{y}) \bar{\varphi}=\frac{\partial L}{\partial y}(x, \bar{y}) \text { in } \Omega, \\
\bar{\varphi}=0 \text { on } \Gamma,
\end{array}\right. \\
& \int_{\Omega}(\bar{\varphi}+\nu \bar{u})(u-\bar{u}) \mathrm{d} x \geq 0 \quad \forall u \in U_{\mathrm{ad}} .
\end{aligned}
$$

This theorem is consequence of the expression for $J^{\prime}$ given in (3.4) and the convexity of $U_{\text {ad }}$, which implies that $J^{\prime}(\bar{u})(u-\bar{u}) \geq 0$ holds for every $u \in U_{\mathrm{ad}}$. As a consequence of this theorem we have the following regularity result on the optimal control.

Corollary 3.7. Let $\bar{u}$ satisfy (3.7)-(3.9) along with $(\bar{y}, \bar{\varphi})$, then $\bar{u} \in H^{1}(\Omega) \cap C(\bar{\Omega})$ holds. Moreover, if $a_{i j} \in$ $C^{0,1}(\bar{\Omega})$ for $1 \leq i, j \leq n, \operatorname{div} b \in L^{2}(\Omega), \bar{p}=2$, and $\Gamma$ is of class $C^{1,1}$ or $\Omega$ is convex, then $\bar{y}, \bar{\varphi} \in H^{2}(\Omega) \cap H_{0}^{1}(\Omega)$ holds. Finally, if $U_{\mathrm{ad}}=L^{2}(\Omega)$, then we have that $\bar{u} \in H^{2}(\Omega) \cap H_{0}^{1}(\Omega)$.

Proof. It is well known that (3.9) implies that

$$
\bar{u}(x)=\operatorname{Proj}_{[\alpha, \beta]}\left(-\frac{1}{\nu} \bar{\varphi}(x)\right) .
$$

Then, the $H^{1}(\Omega) \cap C(\bar{\Omega})$ regularity of $\bar{u}$ follows from this formula and the same regularity of $\bar{\varphi}$. Under the additional assumptions on the data of the problem, the regularity of $\bar{y}$ and $\bar{\varphi}$ follows from Theorem 2.10 and Corollary 2.6. Finally, if $U_{\text {ad }}=L^{2}(\Omega)$, then (3.9) is reduced to $\bar{\varphi}+\nu \bar{u}=0$, hence $\bar{u}$ enjoys the same regularity as $\bar{\varphi}$. 
In order to write the second order optimality conditions we introduce the cone of critical directions. Let $\bar{u} \in U_{\text {ad }}$ be a function satisfying the system (3.7)-(3.9) along with the associated state $\bar{y}$ and adjoint state $\bar{\varphi}$. We define the cone

$$
\begin{gathered}
C_{\bar{u}}=\left\{v \in L^{2}(\Omega): J^{\prime}(\bar{u}) v=0 \text { and (3.10) holds }\right\} \\
v(x, t) \begin{cases}\geq 0 & \text { if } \bar{u}(x, t)=\alpha, \\
\leq 0 & \text { if } \bar{u}(x, t)=\beta .\end{cases}
\end{gathered}
$$

Let us observe that (3.9) implies that

$$
\bar{\varphi}(x)+\nu \bar{u}(x) \begin{cases}\geq 0 & \text { if } \bar{u}(x)=\alpha \\ \leq 0 & \text { if } \bar{u}(x)=\beta\end{cases}
$$

Therefore, if $v \in L^{2}(\Omega)$ satisfies (3.10), then $J^{\prime}(\bar{u}) v \geq 0$ holds, and $J^{\prime}(\bar{u}) v=0$ if and only if $v(x)=0$ if $\bar{\varphi}(x)+$ $\nu \bar{u}(x) \neq 0$.

In the case where there are not control constraints, namely $U_{\text {ad }}=L^{2}(\Omega)$, then $J^{\prime}(\bar{u})=0$ and $C_{\bar{u}}=L^{2}(\Omega)$.

Now, we have the second order conditions.

Theorem 3.8. If $\bar{u}$ is a local solution of $(\mathrm{P})$ in any sense of those given in Definition 3.3, then $J^{\prime \prime}(\bar{u}) v^{2} \geq 0$ $\forall v \in C_{\bar{u}}$. Conversely, if $\bar{u} \in U_{\text {ad }}$ satisfies $(2.12)-(2.14)$ along with $(\bar{y}, \bar{\varphi})$ and

$$
J^{\prime \prime}(\bar{u}) v^{2}>0 \quad \forall v \in C_{\bar{u}} \backslash\{0\}
$$

then there exist $\varepsilon>0$ and $\kappa>0$ such that

$$
J(\bar{u})+\frac{\kappa}{2}\|u-\bar{u}\|_{L^{2}(\Omega)}^{2} \leq J(u) \quad \forall u \in U_{\mathrm{ad}}:\left\|y_{u}-\bar{y}\right\|_{L^{\infty}(\Omega)} \leq \varepsilon .
$$

Proof. The proof follows the steps of [9] or [10]. To reproduce that proof we have to use that $y_{u_{k}} \rightarrow y_{u}$ and $\varphi_{u_{k}} \rightarrow \varphi_{u}$ strongly in $H_{0}^{1}(\Omega) \cap C(\bar{\Omega})$ when $u_{k} \rightarrow u$ in $L^{2}(\Omega)$. The convergence for the states is proved in Theorem 2.11. Here we prove the part corresponding to the adjoint states. To this end we set

$$
\begin{aligned}
& \mathcal{A}^{*} \varphi=A^{*} \varphi-\operatorname{div}[b(x) \varphi]+\frac{\partial f}{\partial y}\left(x, y_{u}\right) \varphi \\
& \mathcal{A}_{k}^{*} \varphi=A^{*} \varphi-\operatorname{div}[b(x) \varphi]+\frac{\partial f}{\partial y}\left(x, y_{u_{k}}\right) \varphi
\end{aligned}
$$

Since $y_{u_{k}} \rightarrow y_{u}$ in $C(\bar{\Omega})$, there exists $M>0$ such that $\left\|y_{u_{k}}\right\|_{C(\bar{\Omega})} \leq M \forall k$. Then, from (2.17) and the mean value theorem we deduce for $\|\varphi\|_{H_{0}^{1}(\Omega)} \leq 1$ :

$$
\begin{aligned}
& \left\|\left(\mathcal{A}^{*}-\mathcal{A}_{k}^{*}\right) \varphi\right\|_{H^{-1}(\Omega)} \leq C_{\Omega}\left\|\left[\frac{\partial f}{\partial y}\left(x, y_{u}\right)-\frac{\partial f}{\partial y}\left(x, y_{u_{k}}\right)\right] \varphi\right\|_{L^{2}(\Omega)} \\
& \leq C_{\Omega} C_{f, M}\left\|y_{u}-y_{u_{k}}\right\|_{C(\bar{\Omega})}\|\varphi\|_{L^{2}(\Omega)} \leq C_{\Omega}^{2} C_{f, M}\left\|y_{u}-y_{u_{k}}\right\|_{C(\bar{\Omega})} \stackrel{k \rightarrow \infty}{\longrightarrow} 0 .
\end{aligned}
$$

Hence, we can proceed as in the proof of Theorem 2.5 to deduce the existence of $k_{0}$ such that

$$
\left\|\left[\mathcal{A}_{k}^{*}\right]^{-1}\right\|_{\mathcal{L}\left(H^{-1}(\Omega), H_{0}^{1}(\Omega)\right)} \leq 2\left\|\left[\mathcal{A}^{*}\right]^{-1}\right\|_{\mathcal{L}\left(H^{-1}(\Omega), H_{0}^{1}(\Omega)\right)} \quad \forall k \geq k_{0} .
$$


In addition, arguing as in Step 2 of the proof of Theorem 2.5, we have

$$
\left\|\left[\mathcal{A}^{*}\right]^{-1}-\left[\mathcal{A}_{k}^{*}\right]^{-1}\right\|_{\mathcal{L}\left(H^{-1}(\Omega), H_{0}^{1}(\Omega)\right)} \rightarrow 0 \quad \text { when } k \rightarrow \infty
$$

Hence, we get with (3.2)

$$
\begin{aligned}
& \left\|\varphi_{u}-\varphi_{u_{k}}\right\|_{H_{0}^{1}(\Omega)}=\left\|\left[\mathcal{A}^{*}\right]^{-1} \frac{\partial L}{\partial y}\left(x, y_{u}\right)-\left[\mathcal{A}_{k}^{*}\right]^{-1} \frac{\partial L}{\partial y}\left(x, y_{u_{k}}\right)\right\|_{H_{0}^{1}(\Omega)} \\
& \leq\left\|\left(\left[\mathcal{A}^{*}\right]^{-1}-\left[\mathcal{A}_{k}^{*}\right]^{-1}\right) \frac{\partial L}{\partial y}\left(x, y_{u}\right)\right\|_{H_{0}^{1}(\Omega)} \\
& \quad+\left\|\left[\mathcal{A}_{k}^{*}\right]^{-1}\left[\frac{\partial L}{\partial y}\left(x, y_{u}\right)-\frac{\partial L}{\partial y}\left(x, y_{u_{k}}\right)\right]\right\|_{H_{0}^{1}(\Omega)} \\
& \leq\left\|\left[\mathcal{A}^{*}\right]^{-1}-\left[\mathcal{A}_{k}^{*}\right]^{-1}\right\|_{\mathcal{L}\left(H^{-1}(\Omega), H_{0}^{1}(\Omega)\right)}\left\|\frac{\partial L}{\partial y}\left(x, y_{u}\right)\right\|_{H^{-1}(\Omega)} \\
& \quad+2\left\|\left[\mathcal{A}^{*}\right]^{-1}\right\|_{\mathcal{L}\left(H^{-1}(\Omega), H_{0}^{1}(\Omega)\right)}\left\|\frac{\partial L}{\partial y}\left(x, y_{u}\right)-\frac{\partial L}{\partial y}\left(x, y_{u_{k}}\right)\right\|_{H^{-1}(\Omega)} \\
& \leq\left\|\left[\mathcal{A}^{*}\right]^{-1}-\left[\mathcal{A}_{k}^{*}\right]^{-1}\right\|_{\mathcal{L}\left(H^{-1}(\Omega), H_{0}^{1}(\Omega)\right)}\left\|\frac{\partial L}{\partial y}\left(x, y_{u}\right)\right\|_{H^{-1}(\Omega)} \\
& \quad+2 C_{\Omega} C_{L, M}\left\|\left[\mathcal{A}^{*}\right]^{-1}\right\|_{\mathcal{L}\left(H^{-1}(\Omega), H_{0}^{1}(\Omega)\right)}\left\|y_{u}-y_{u_{k}}\right\|_{L^{2}(\Omega)} \rightarrow 0 .
\end{aligned}
$$

It remains to prove that $\left\|\varphi_{u}-\varphi_{u_{k}}\right\|_{C(\bar{\Omega})} \rightarrow 0$. The equation satisfied by $\varphi_{u_{k}}$ can be written as

$$
A^{*} \varphi_{u_{k}}-\operatorname{div}\left(b(x) \varphi_{u_{k}}\right)=-\frac{\partial f}{\partial y}\left(x, y_{u_{k}}\right) \varphi_{u_{k}}+\frac{\partial L}{\partial y}\left(x, y_{u_{k}}\right) .
$$

Since $\left\{\varphi_{u_{k}}\right\}_{k=1}^{\infty}$ is convergent in $H_{0}^{1}(\Omega)$, we have that it is bounded in $L^{2}(\Omega)$. Using this, the fact that $\left\|y_{u_{k}}\right\|_{C(\bar{\Omega})} \leq$ $M$, and Assumptions 3 and 4 , we have that the right hand side is bounded in $L^{\bar{p}}(\Omega)$. Hence from Corollary 2.4 we deduce that $\left\{\varphi_{u_{k}}\right\}_{k=1}^{\infty}$ is bounded in $C^{0, \mu}(\bar{\Omega})$ for some $\mu>0$. Then, the convergence in $C(\bar{\Omega})$ follows from the compact embedding $C^{0, \mu}(\bar{\Omega}) \subset C(\bar{\Omega})$.

The following corollary is an immediate consequence of Theorem 3.8 and Lemma 3.5.

Corollary 3.9. Under the assumptions of Theorem 3.8. there exist $\kappa>0$ and $\varepsilon>0$ such that

$$
J(\bar{u})+\frac{\kappa}{2}\|u-\bar{u}\|_{L^{2}(\Omega)}^{2} \leq J(u) \quad \forall u \in U_{\mathrm{ad}}:\|u-\bar{u}\|_{L^{2}(\Omega)} \leq \varepsilon
$$

It is interesting to remark that if $\bar{u}$ satisfies (3.7)-(3.9) and (3.11), then besides of being a strict local solution of $(\mathrm{P})$, there exists a ball $B_{r}(\bar{u}) \subset L^{2}(\Omega)$ such that $\bar{u}$ is the unique stationary point of $(\mathrm{P})$ in $B_{r}(\bar{u})$, i.e. the unique control satisfying (3.7)-(3.9); see Corollary 2.6 from [9].

\section{REFERENCES}

[1] H.T. Banks and K. Kunisch, Estimation techniques for distributed parameter systems, volume 1 of Systems 8 Control: Foundations \&J Applications. Birkhäuser Boston, Inc., Boston, MA (1989).

[2] T. Bayen and F. Silva, Second order analysis for strong solutions in the optimal control of parabolic equations. SIAM J. Control Optim. 54 (2016) 819-844.

[3] T. Bayen, F. Bonnans and F. Silva, Characterization of local quadratic growth for strong minima in the optimal control of semi-linear elliptic equations. Trans. Amer. Math. Soc. 366 (2014) 2063-2087.

[4] L. Boccardo, Stampacchia-Caldéron-Zygmund theory for linear elliptic equations with discontinuous coefficients and singular drift. ESAIM: COCV 25 (2019) 47. 
[5] L. Boccardo, Two semilinear Dirichlet problems "almost" in duality. Boll. Unione Mat. It. 12 (2019) 349-356.

[6] E. Casas and M. Mateos, Optimal control of partial differential equations, in Computational mathematics, numerical analysis and applications, Vol. 13 of SEMA SIMAI Springer Series. Springer, Cham (2017) 3-59.

[7] E. Casas and M. Mateos, Critical cones for sufficient second order conditions in PDE constrained optimization. SIAM J. Optim. 30 (2020) 585-603.

[8] E. Casas and F. Tröltzsch, Optimality conditions for a class of optimal control problems with quasilinear elliptic equations. SIAM J. Control Optim. 48 (2009) 688-718.

[9] E. Casas and F. Tröltzsch, Second order analysis for optimal control problems: Improving results expected from abstract theory. SIAM J. Optim. 22 (2012) 261-279.

[10] E. Casas and F. Tröltzsch, Second order optimality conditions and their role in PDE control. Jahresber Dtsch Math-Ver $\mathbf{1 1 7}$ (2015) 3-44.

[11] E. Casas and F. Tröltzsch, State-constrained semilinear elliptic optimization problems with unrestricted sparse controls. Math. Control Relat. Fields 10 (2020) 527-546.

[12] L.C. Evans, Partial differential equations, Vol. 19 of Graduate Studies in Mathematics. American Mathematical Society, Providence, RI (1998).

[13] D. Gilbarg and N.S. Trudinger, Elliptic Partial Differential Equations of Second Order. Springer-Verlag, Berlin (1983).

[14] P. Grisvard, Elliptic Problems in Nonsmooth Domains. Pitman, Boston (1985).

[15] M. Kř́žzek and L. Liu, On the maximum and comparison pronciples for a steady-state nonlinear heat condution problem. $Z$. Angew. Math. Mech. 83 (2003) 559-563.

[16] O.A. Ladyzhenskaya and N.N. Ural'tseva, Linear and quasilinear elliptic equations. Translated from the Russian by Scripta Technica, Inc. Translation editor: Leon Ehrenpreis. Academic Press, New York (1968).

[17] J. Nečas, Les Méthodes Directes en Théorie des Equations Elliptiques. Editeurs Academia, Belgium (1967).

[18] G. Stampacchia, Le problème de Dirichlet pour les équations elliptiques du second ordre à coefficients discontinus. Ann. Inst. Fourier (Grenoble) 15 (1965) 189-258.

[19] N.S. Trudinger, Linear elliptic operators with measurable coefficients. Ann. Scuola Norm. Sup. Pisa Cl. Sci. 27 (1973) $265-308$. 